\title{
SHARP ESTIMATES FOR THE SZEGÖ PROJECTION ON THE DISTINGUISHED BOUNDARY OF MODEL WORM DOMAINS
}

\author{
ALESSANDRO MONGUZZI AND MARCO M. PELOSO
}

\begin{abstract}
In this paper we study the regularity of the Szegö projection on Lebesgue and Sobolev spaces on the distinguished boundary of the unbounded model worm domain $D_{\beta}$.

We denote by $d_{b}\left(D_{\beta}\right)$ the distinguished boundary of $D_{\beta}$ and define the corresponding Hardy space $\mathscr{H}^{2}\left(D_{\beta}\right)$. This can be identified with a closed subspace of $L^{2}\left(d_{b}\left(D_{\beta}\right), d \sigma\right)$, that we denote by $\mathscr{H}^{2}\left(d_{b}\left(D_{\beta}\right)\right)$, where $d \sigma$ is the naturally induced measure on $d_{b}\left(D_{\beta}\right)$.

The orthogonal Hilbert space projection $\mathscr{P}: L^{2}\left(d_{b}\left(D_{\beta}\right), d \sigma\right) \rightarrow \mathscr{H}^{2}\left(d_{b}\left(D_{\beta}\right)\right)$ is called the Szegő projection on the distinguished boundary.

We prove that $\mathscr{P}$, initially defined on the dense subspace $L^{2}\left(d_{b}\left(D_{\beta}\right), d \sigma\right) \cap L^{p}\left(d_{b}\left(D_{\beta}\right), d \sigma\right)$ extends to a bounded operator $\mathscr{P}: L^{p}\left(d_{b}\left(D_{\beta}\right), d \sigma\right) \rightarrow L^{p}\left(d_{b}\left(D_{\beta}\right), d \sigma\right)$ if and only if $\frac{2}{1+\nu_{\beta}}<p<$ $\frac{2}{1-\nu_{\beta}}$ where $\nu_{\beta}=\frac{\pi}{2 \beta-\pi}, \beta>\pi$. Furthermore, we also prove that $\mathscr{P}$ defines a bounded operator $\mathscr{P}: W^{s, 2}\left(d_{b}\left(D_{\beta}\right), d \sigma\right) \rightarrow W^{s, 2}\left(d_{b}\left(D_{\beta}\right), d \sigma\right)$ if and only if $0 \leq s<\frac{\nu_{\beta}}{2}$ where $W^{s .2}\left(d_{b}\left(D_{\beta}\right), d \sigma\right)$ denotes the Sobolev space of order $s$ and underlying $L^{2}$-norm.

Finally, we prove a necessary condition for the boundedness of $\mathscr{P}$ on $W^{s, p}\left(d_{b}\left(D_{\beta}\right), d \sigma\right)$, $p \in(1, \infty)$, the Sobolev space of order $s$ and underlying $L^{p}$-norm.
\end{abstract}

\section{Introduction AND STATEMEnt of the main RESUlts}

In this paper we consider the Hardy spaces on the distinguished boundary of the model worm domain

$$
D_{\beta}=\left\{\left(z_{1}, z_{2}\right) \in \mathbb{C}^{2}: \operatorname{Re}\left(z_{1} e^{-i \log \left|z_{2}\right|^{2}}\right)>0,\left.|\log | z_{2}\right|^{2} \mid<\beta-\frac{\pi}{2}\right\}, \quad \beta>\pi,
$$

and study the continuity of the associated Szegő projection operator on the Lebesgue and Sobolev spaces.

The domain $D_{\beta}$ is unbounded, pseudoconvex, with Lipschitz boundary, and was instrumental in proving that the Bergman projection does not preserve Sobolev spaces of sufficiently high order on the smooth pseudoconvex worm domain $\Omega_{\beta}$ introduced by K. Diederich and J.E. Fornæss [DF77]. This result, due to D. Barrett [Bar92], was a major breakthrough since it suggested that some long-standing conjectures about the geometry of smooth pseudoconvex domains and the regularity of the associated Bergman projection were actually false. Indeed, few years later, M. Christ Chr96] proved that the Bergman projection on the Diederich-Fornæss worm domain does not preserve the space $C^{\infty}\left(\overline{\Omega_{\beta}}\right)$ - that is, Condition $R$ fails on $\Omega_{\beta}$. We refer the reader to KP08a for a detailed account on the subject.

2010 Mathematics Subject Classification. 32A25, 32A36, 30H20.

Key words and phrases. Hardy spaces, Szegő kernel, Szegő projection, worm domain.

Both authors supported in part by the 2010-11 PRIN grant Real and Complex Manifolds: Geometry, Topology and Harmonic Analysis of the Italian Ministry of Education (MIUR). 
The question of the regularity of the Bergman projection on worm domains and related questions has been considered by various authors, and here we mention in particular [KP08b, BŞ12, BEP15, KPS15, KPS16, CŞ15.

On worms domains, of course it is of great interest also to study the (ir-)regularity of the boundary analogue of the Bergman projection, that is, the Szegő projection. If $\Omega=\{z: \rho(z)<$ $0\}$ is a smoothly bounded domain in $\mathbb{C}^{n}$, the Hardy space $H^{2}(\Omega)$ is defined as

$$
H^{2}(\Omega)=\left\{f \in \operatorname{Hol}(\Omega): \sup _{\varepsilon>0} \int_{b \Omega_{\varepsilon}}|f|^{2} d \sigma_{\varepsilon}<\infty\right\},
$$

where $\Omega_{\varepsilon}=\{z: \rho(z)<-\varepsilon\}$ and $d \sigma_{\varepsilon}$ is the induced surface measure on $b \Omega_{\varepsilon}$. Here, and in what follows, we denote by $b \mathcal{D}$ the topological boundary of a given domain $\mathcal{D}$. Then, $H^{2}(\Omega)$ can be identified with a closed subspace of $L^{2}(b \Omega, d \sigma)$, that we denote by $H^{2}(b \Omega)$. The Szegö projection is the orthogonal projection

$$
P_{\Omega}: L^{2}(b \Omega, d \sigma) \rightarrow H^{2}(b \Omega) ;
$$

see [Ste70]. Mapping properties of the Szegö projection on other function spaces have been studied for various classes of smooth bounded domains. In the case of strictly pseudoconvex domains [PS77, domains of finite type in $\mathbb{C}^{2}$ [NRSW89], convex domains of finite type in $\mathbb{C}^{n}$ [MS97] the Szegó projection $P_{\Omega}$ turns out to be bounded on the Lebesgue-Sobolev spaces $W^{s, p}(b \Omega)$ for $1<p<\infty$ and $s \geq 0$. When $\Omega$ is Reinhardt domain Boa85, Str86, a domain with partially transverse symmetries [BCS88], a pseudoconvex domain satisfying Catlin's property $(\mathcal{P})$ [Boa87, a complete Hartogs domain in $\mathbb{C}^{2}$ [BS89], or a domain with a plurisubharmonic defining function on the boundary BS91, then the Szego" projection is exactly regular, that is, $P_{\Omega}$ is a bounded operator $P_{\Omega}: W^{s, 2}(b \Omega) \rightarrow W^{s, 2}(b \Omega)$ for every $s \geq 0$. We also mention that, if $\Omega$ is bounded, $C^{2}$ and strongly pseudoconvex in $\mathbb{C}^{n}$, the Szegö projection $P_{\Omega}$ again extends to bounded operator on $L^{p}(b \Omega)$ for $1<p<\infty$, LS15a, LS15b].

There are examples of domains $\Omega$ on which the Szegö projection $P_{\Omega}$ is less regular. L. Lanzani and E.M. Stein described the (ir-)regularity of $P_{\Omega}$ on Lebesgue spaces in the case of planar simply connected domains, [LS04, Thm. 2.1]. In particular they showed that if $\Omega$ has Lipschitz boundary, then $P_{\Omega}: L^{p}(b \Omega) \rightarrow L^{p}(b \Omega)$ if and only if $p_{\Omega}^{\prime}<p<p_{\Omega}$, where $p_{\Omega}$ depends only on the Lipschitz constant of $b \Omega$. More recently, S. Munasinghe and Y.E. Zeytuncu provided an example of a piecewise smooth, bounded pseudoconvex domain in $\mathbb{C}^{2}$ on which the Szegö projection $P_{\Omega}$ is unbounded on $L^{p}(b \Omega)$ for every $p \neq 2$ [MZ15. The same result on tube domains over irriducible self-dual cones of rank greater than 1 has been known for a number of years, BB95].

Clearly the Szegö projection depends on the choice of the measure on the boundary. For instance, instead of taking the induced surface measure $d \sigma$, one can consider the Fefferman surface measure, see [BL14, or any surface measure of the form $\omega d \sigma$, with $\omega$ continuous and positive, [LS15b]. On non-smooth domains, such as the polydisk, it is also of interest, and perhaps more natural, to study Hardy spaces defined by integration over the so-called distinguished boundary. For a general domain $\Omega \subseteq \mathbf{C}^{n}$, the distinguished boundary $d_{b}(\Omega)$ is the set

$$
d_{b}(\Omega)=\left\{\zeta \in b \Omega: \sup _{z \in b \Omega}|f(z)| \leq \sup _{\zeta \in d_{b}(\Omega)}|f(\zeta)| \text { for all } f \in H^{\infty}(\Omega)\right\}
$$

where $H^{\infty}(\Omega)$ denotes the spaces of bounded holomorphic functions on $\Omega$.

In this paper we obtain sharp results concerning the regularity of the Szegö projection $\mathscr{P}=$ $\mathscr{P}_{D_{\beta}}$ for the Hardy spaces on the distinguished boundary of the model worm domain $D_{\beta}$, that 
is

$$
d_{b}\left(D_{\beta}\right)=\left\{\left(z_{1}, z_{2}\right) \in \mathbb{C}^{2}:\left.\left|\arg z_{1}-\log \right| z_{2}\right|^{2}\left|=\frac{\pi}{2},\right| \log \left|z_{2}\right|^{2} \mid=\beta-\frac{\pi}{2}\right\} .
$$

Our final goal is to study the (ir-)regularity of the Szegö projection of the smooth worm $\Omega_{\beta}$ and the present work is a step in this direction. As is the Bergman setting, it is reasonable to expect that the peculiar geometry of the worm domain affects the regularity of the Szegö projection as well. The results in this paper support this expectancy.

In shifting from the Bergman to the Szegö setting new difficulties arise and Barrett's arguments cannot be trivially adapted to transfer information from the model worm $D_{\beta}$ to the smooth worm $\Omega_{\beta}$ [Mon16c]. Barrett proved the irregularity of the Bergman projection $B_{D_{\beta}}$ on $W^{s, 2}\left(\Omega_{\beta}\right)$ if $s \geq \pi /(2 \beta-\pi)$ by means of the well-known transformation rule for the Bergman projection and by studying the Bergman projection of a biholomorphic copy of $D_{\beta}$, classically denoted by $D_{\beta}^{\prime}$. Subsequently, if $\Omega_{\beta, \lambda}, \lambda>0$, denotes an appropriate dilation of $\Omega_{\beta}$, Barrett proved that $B_{\Omega_{\beta, \lambda}} \rightarrow B_{D_{\beta}}$ as $\lambda \rightarrow \infty$ in a suitable sense. Thus, if $B_{\Omega_{\beta}}$ were bounded on $W^{s, 2}\left(\Omega_{\beta}\right)$, then also $B_{D_{\beta}}$ would be bounded on $W^{s, 2}\left(D_{\beta}\right)$. Hence, by contradiction, it follows that $B_{\Omega_{\beta}}$ cannot be bounded on $W^{s, 2}\left(\Omega_{\beta}\right)$ if $s \geq \pi /(2 \beta-\pi)$.

In the Szegö setting, in general, there is no transformation rule for the Szegö projection under biholomorphic mappings. Nonetheless, we are able to prove a transformation rule for the projections $\mathscr{P}$ and $\mathscr{P}^{\prime}=\mathscr{P}_{D_{\beta}^{\prime}}$, see Section [6. The mapping properties of the Szegö projection $\mathscr{P}^{\prime}$ on the distinguished boundary of $D_{\beta}^{\prime}$ were studied by the first author in Mon16b, Mon16a].

We now describe our results in greater details. For $(t, s) \in\left(0, \frac{\pi}{2}\right) \times\left[0, \beta-\frac{\pi}{2}\right)$ consider the domain

$$
D_{t, s}=\left\{\left(z_{1}, z_{2}\right) \in \mathbb{C}^{2}:\left.\left|\arg z_{1}-\log \right| z_{2}\right|^{2}|<t,| \log \left|z_{2}\right|^{2} \mid<s\right\} .
$$

Then, the collection $\left\{D_{t, s}\right\}_{t, s}$ is a family of approximating domains for $D_{\beta}$ and the distinguished boundary of each of these domains is

$$
d_{b}\left(D_{t, s}\right)=\left\{\left(z_{1}, z_{2}\right) \in \mathbb{C}^{2}:\left.\left|\arg z_{1}-\log \right| z_{2}\right|^{2}|=t,| \log \left|z_{2}\right|^{2} \mid=s\right\} .
$$

Consequently, for $1 \leq p<\infty$, we define the Hardy space $\mathscr{H}^{p}\left(D_{\beta}\right)$ as

$$
\mathscr{H}^{p}\left(D_{\beta}\right):=\left\{f \in \operatorname{Hol}\left(D_{\beta}\right):\|f\|_{\mathscr{H}^{p}\left(D_{\beta}\right)}^{p}=\sup _{(t, s) \in\left(0, \frac{\pi}{2}\right) \times\left[0, \beta-\frac{\pi}{2}\right)}\|f\|_{L^{p}\left(d_{b}\left(D_{t, s}\right)\right)}^{p}<\infty\right\},
$$

where, denoting by $d \sigma_{t, s}$ the induced measure on $d_{b}\left(D_{t, s}\right)$,

$$
\begin{aligned}
& \|f\|_{L^{p}\left(d_{b}\left(D_{t, s}\right)\right)}^{p}=\int_{d_{b}\left(D_{t, s}\right)}|f|^{p} d \sigma_{t, s} \\
& =\int_{0}^{\infty} \int_{0}^{2 \pi}\left|f\left(r e^{i(s+t)}, e^{\frac{s}{2}} e^{i \theta}\right)\right|^{p} e^{\frac{s}{2}} d \theta d r+\int_{0}^{\infty} \int_{0}^{2 \pi}\left|f\left(r e^{i(s-t)}, e^{\frac{s}{2}} e^{i \theta}\right)\right|^{p} e^{\frac{s}{2}} d \theta d r \\
& \quad+\int_{0}^{\infty} \int_{0}^{2 \pi}\left|f\left(r e^{-i(s+t)}, e^{-\frac{s}{2}} e^{i \theta}\right)\right|^{p} e^{-\frac{s}{2}} d \theta d r+\int_{0}^{\infty} \int_{0}^{2 \pi}\left|f\left(r e^{-i(s-t)}, e^{-\frac{s}{2}} e^{i \theta}\right)\right|^{p} e^{-\frac{s}{2}} d \theta d r .
\end{aligned}
$$

In Section 3 we will discuss the above definition and show that it is a very natural one. 
Standard basic facts of Hardy space theory give that any $f \in \mathscr{H}^{p}$ admits a boundary value function, that we still denote by $f$, defined on $d_{b}\left(D_{\beta}\right)$ that is $p$-integrable w.r.t. $d \sigma$, the induced surface measure on $d_{b}\left(D_{\beta}\right)$. Moreover, we have the equality

$$
\|f\|_{\mathscr{H}^{p}\left(D_{\beta}\right)}^{p}=\int_{d_{b}\left(D_{\beta}\right)}|f|^{p} d \sigma .
$$

Then, we can identify $\mathscr{H}^{p}$ with a subspace of $L^{p}\left(d_{b}\left(D_{\beta}\right), d \sigma\right)=L^{p}\left(d_{b}\left(D_{\beta}\right)\right)$, that is closed and that we denote by $\mathscr{H}^{p}\left(d_{b}\left(D_{\beta}\right)\right)$. The Szegó projection $\mathscr{P}$ is the the orthogonal projection of $L^{2}\left(d_{b}\left(D_{\beta}\right)\right)$ onto $\mathscr{H}^{2}\left(d_{b}\left(D_{\beta}\right)\right)$

$$
\mathscr{P}: L^{2}\left(d_{b}\left(D_{\beta}\right)\right) \rightarrow \mathscr{H}^{2}\left(d_{b}\left(D_{\beta}\right)\right) .
$$

The operator $\mathscr{P}$ admits an integral representation and we call its integral kernel the Szegö kernel. It turns out that in this case the operator $\mathscr{P}$ can be written as a combination of Mellin-Fourier multiplier operators (see Section 2). Our main results are the following.

Theorem 1. Let be $\nu_{\beta}=\frac{\pi}{2 \beta-\pi}$. Then, the Szegö projection $\mathscr{P}$, initially defined on the dense subspace $L^{p}\left(\partial D_{\beta}\right) \cap L^{2}\left(d_{b}\left(D_{\beta}\right)\right)$, extends to a bounded operator

$$
\mathscr{P}: L^{p}\left(d_{b}\left(D_{\beta}\right)\right) \rightarrow L^{p}\left(d_{b}\left(D_{\beta}\right)\right)
$$

if and only if $\frac{2}{1+\nu_{\beta}}<p<\frac{2}{1-\nu_{\beta}}$.

Theorem 2. Let be $\nu_{\beta}=\frac{\pi}{2 \beta-\pi}$. Then, the Szegö projection $\mathscr{P}$ defines a bounded operator

$$
\mathscr{P}: W^{s, 2}\left(d_{b}\left(D_{\beta}\right)\right) \rightarrow W^{s, 2}\left(d_{b}\left(D_{\beta}\right)\right)
$$

if and only if $0 \leq s<\frac{\nu_{\beta}}{2}$.

Our results thus completely describes the mapping properties of the operator $\mathscr{P}$ with respect to the Lebesgue and Sobolev-Hilbert norms. If we consider Sobolev norms with $p \neq 2$ we do not have a complete characterization of the mapping properties of $\mathscr{P}$, but we have a partial result.

Theorem 3. Let be $\nu_{\beta}=\frac{\pi}{2 \beta-\pi}, s>0$ and $p \in(1, \infty)$. If the operator $\mathscr{P}$, initially defined on the dense subspace $W^{s, p}\left(d_{b}\left(D_{\beta}\right)\right) \cap L^{2}\left(d_{b}\left(D_{\beta}\right)\right)$, extends to a bounded operator $\mathscr{P}: W^{s, p}\left(d_{b}\left(D_{\beta}\right)\right) \rightarrow$ $W^{s, p}\left(d_{b}\left(D_{\beta}\right)\right)$, then

$$
-\frac{\nu_{\beta}}{2} \leq s+\frac{1}{2}-\frac{1}{p} \leq \frac{\nu_{\beta}}{2}
$$

Assuming $p \geq 2$ we obtain the stronger condition

$$
0 \leq s+\frac{1}{2}-\frac{1}{p}<\frac{\nu_{\beta}}{2}
$$

In this work, $W^{s, p}\left(d_{b}\left(D_{\beta}\right)\right)$ for a non-integer $s$ denotes the classical fractional Sobolev space defined via the Fourier transform and the Bessel potentials. We refer the reader to Section 4.1 for the precise definition.

The paper is organized as follows. In Section 2 we introduce Mellin-Fourier multiplier operators and we provide a sufficient condition for their $L^{p}$ continuity. In Section 3 we study the space $\mathscr{H}^{2}\left(D_{\beta}\right)$, we show that the Szegö projection $\mathscr{P}$ is given by a sum of Mellin-Fourier multiplier operators and we prove the sufficient condition of Theorem 1. In Section 4 we conclude the proof of Theorem 1 and we prove the Sobolev irregularity, whereas in Section 5 we prove the 
Sobolev regularity. In Section 6 we prove the transformation rule for $\mathscr{P}$ and $\mathscr{P}$. We conclude the paper with some final remarks.

\section{Mellin-Fourier Multiplier operators}

In this section we introduce a class of operators, which we call Mellin-Fourier multiplier operators, and provide a sufficient condition for their $L^{p}$ continuity, $p \in(1, \infty)$. A similar class of operators was studied by Rooney [Ro085]. Incidentally, we believe that this class of operators is of its own interest.

We are going to work both on $\mathbb{R}^{2}$ and on $\mathbb{R} \times \mathbb{T}$, where $\mathbb{T}=\mathbb{R} / \mathbb{Z}$ is the torus. Thus, we set $\mathbb{X}$ to denote either $\mathbb{R}$ or $\mathbb{T}$, and, accordingly, $\widehat{\mathbb{X}}=\mathbb{R}$, or $\mathbb{Z}$, respectively. This allows us to unify the presentation and should cause no confusion.

We denote by $\mathcal{F}$ the Fourier transform on $\mathbb{R} \times \mathbb{X}$, given by

$$
\mathcal{F} f\left(\xi_{1}, \xi_{2}\right)=\int_{\mathbb{R} \times \mathbb{X}} f\left(x_{1}, x_{2}\right) e^{-i\left(x_{1} \xi_{+} x_{2} \xi_{2}\right)} d x_{1} d x_{2}
$$

when $f$ is absolutely integrable, and by $T_{m}$ the Fourier multiplier operator

$$
T_{m}(f)=\mathcal{F}^{-1}(m \widehat{f})
$$

when $m$ is a bounded measurable function on $\mathbb{R} \times \widehat{\mathbb{X}}$, where we also write $\mathcal{F} f=\widehat{f}$. We say that a bounded function $m$ on $\mathbb{R} \times \mathbb{X}$ is a bounded Fourier multiplier on $L^{p}(\mathbb{R} \times \mathbb{X})$ if $T_{m}: L^{p}(\mathbb{R} \times \mathbb{X}) \rightarrow$ $L^{p}(\mathbb{R} \times \mathbb{X})$ is bounded.

For a function $\varphi \in C_{c}^{\infty}(0, \infty)$ we define the operator $\mathcal{C}_{p}$

$$
\mathcal{C}_{p} \varphi(x)=e^{\frac{x}{p}} \varphi\left(e^{x}\right) .
$$

Clearly $\mathcal{C}_{p}$ extends to an isometry of $L^{p}((0,+\infty))$ onto $L^{p}(\mathbb{R})$. With an abuse of notation, for a fixed $\alpha \in \mathbb{R}$, we also denote by $\mathcal{C}_{p}$ the operator defined on functions in $C_{c}^{\infty}\left((0,+\infty) e^{i \alpha} \times \mathbb{X}\right)$ and acting on the first variable only, that is,

$$
\mathcal{C}_{p} \varphi(x+i \alpha, y)=e^{\frac{1}{p}(x+i \alpha)} \varphi\left(e^{x+i \alpha}, y\right) .
$$

For $a, b \in \mathbb{R}$, with $0<a<b<1$, we denote by $S_{a, b}$ the vertical strip in the complex plane

$$
S_{a, b}=\{z \in \mathbb{C}: a<\operatorname{Re} z<b\} .
$$

Given a bounded measurable function $m$ defined on $S_{a, b} \times \mathbb{X}$, when $a<\frac{1}{p}<b$ we write

$$
m_{p}\left(\xi_{1}, \xi_{2}\right)=m\left(\frac{1}{p}-i \xi_{1}, \xi_{2}\right) .
$$

Finally, we define an operator acting on functions defined on $(0,+\infty) \times \mathbb{X}$ as

$$
\mathscr{T}_{m, p}=\mathcal{C}_{p}^{-1} T_{m_{p}} \mathcal{C}_{p}
$$

We call such an operator a Mellin-Fourier multiplier operator, the reason for which will soon be clear.

Theorem 2.1. With the above notation, let $m: S_{a, b} \times \mathbb{X} \rightarrow \mathbb{C}$ be continuous and such that

(i) $m\left(\cdot, \xi_{2}\right) \in \operatorname{Hol}\left(S_{a, b}\right)$ and bounded in every closed substrip of $S_{a, b}$, for every $\xi_{2} \in \mathbb{X}$ fixed;

(ii) for every $q$ such that $a<\frac{1}{q}<b, m_{q}$ is a bounded Fourier multiplier on $L^{q}(\mathbb{R} \times \mathbb{X})$. 
Then, for $a<\frac{1}{p}<b, \mathscr{T}_{m, p}=\mathscr{T}_{m}$ is independent of $p$ and

$$
\mathscr{T}_{m}: L^{p}((0,+\infty) \times \mathbb{X}) \rightarrow L^{p}((0,+\infty) \times \mathbb{X})
$$

is bounded.

Proof. The fact that $\mathscr{T}_{m, p}: L^{p}((0,+\infty) \times \mathbb{X}) \rightarrow L^{p}((0,+\infty) \times \mathbb{X})$ is bounded is clear since for $\varphi \in C_{c}^{\infty}((0,+\infty) \times \mathbb{X})$

$$
\begin{aligned}
\left\|\mathscr{T}_{m, p}(\varphi)\right\|_{L^{p}((0,+\infty) \times \mathbb{X})} & =\left\|T_{m_{p}} \mathcal{C}_{p}(\varphi)\right\|_{L^{p}(\mathbb{R} \times \mathbb{X})} \\
& \leq\left\|m_{p}\right\|_{\mathcal{M}_{p}(\mathbb{R} \times \mathbb{X})}\left\|\mathcal{C}_{p}(\varphi)\right\|_{L^{p}(\mathbb{R} \times \mathbb{X})} \\
& =\left\|m_{p}\right\|_{\mathcal{M}_{p}(\mathbb{R} \times \mathbb{X})}\|\varphi\|_{L^{p}((0,+\infty) \times \mathbb{X})} .
\end{aligned}
$$

Here we denote by $\left\|m_{p}\right\|_{\mathcal{M}_{p}(\mathbb{R} \times \mathbb{X})}$ the operator norm of the bounded Fourier multiplier $m$ on $L^{p}(\mathbb{R} \times \mathbb{X})$.

Thus, it suffices to show that $\mathscr{T}_{m, p}$ is independent of $p$, when $a<\frac{1}{p}<b$. We show the argument in the case $\mathbb{X}=\mathbb{R}$ since the case $\mathbb{X}=\mathbb{T}$ is identical, one only has to replace integration on $\mathbb{R}$ w.r.t. to $d \xi_{2}$ by a summation over $\mathbb{Z}$.

Setting $g=\mathcal{C}_{p}(\varphi)$ we observe that, for $\left.\left(t, x_{2}\right) \in(0,+\infty) \times \mathbb{X}\right)$, by Fubini's theorem,

$$
\begin{aligned}
& \mathcal{C}_{p}^{-1} T_{m_{p}} \mathcal{C}_{p}(\varphi)\left(t, x_{2}\right) \\
& =t^{-\frac{1}{p}} T_{m_{p}} g\left(\log t, x_{2}\right)=t^{-\frac{1}{p}} \mathcal{F}^{-1}\left(m_{p} \widehat{g}\right)\left(\log t, x_{2}\right) \\
& =\frac{1}{(2 \pi)^{2}} t^{-\frac{1}{p}} \int_{\mathbb{R} \times \mathbb{R}} e^{i\left(\xi_{1} \log t+\xi_{2} x_{2}\right)} m\left(\frac{1}{p}-i \xi_{1}, \xi_{2}\right) \widehat{g}\left(\xi_{1}, \xi_{2}\right) d \xi_{1} d \xi_{2} \\
& =\frac{1}{(2 \pi)^{2}} \int_{\mathbb{R}} \int_{\mathbb{R}} t^{-\frac{1}{p}+i \xi_{1}} m\left(\frac{1}{p}-i \xi_{1}, \xi_{2}\right) \widehat{g}\left(\xi_{1}, \xi_{2}\right) d \xi_{1} e^{i \xi_{2} x_{2}} d \xi_{2} \\
& =\frac{1}{(2 \pi)^{2}} \int_{\mathbb{R}} \int_{\mathbb{R}} t^{-\frac{1}{p}+i \xi_{1}} m\left(\frac{1}{p}-i \xi_{1}, \xi_{2}\right) \int_{\mathbb{R}^{2}} e^{-i\left(y_{1} \xi_{1}+y_{2} \xi_{2}\right)} g\left(y_{1}, y_{2}\right) d y_{1} d y_{2} d \xi_{1} e^{i \xi_{2} x_{2}} d \xi_{2} \\
& =\frac{1}{(2 \pi)^{2}} \int_{\mathbb{R}} \int_{\mathbb{R}} t^{-\frac{1}{p}+i \xi_{1}} m\left(\frac{1}{p}-i \xi_{1}, \xi_{2}\right) \int_{\mathbb{R}^{2}} e^{-i\left(y_{1} \xi_{1}+y_{2} \xi_{2}\right)} e^{\frac{y_{1}}{p}} \varphi\left(e^{y_{1}}, y_{2}\right) d y_{1} d y_{2} d \xi_{1} e^{i \xi_{2} x_{2}} d \xi_{2} \\
& =\frac{1}{(2 \pi)^{2}} \int_{\mathbb{R}} \int_{\mathbb{R}} t^{-\frac{1}{p}+i \xi_{1}} m\left(\frac{1}{p}-i \xi_{1}, \xi_{2}\right) \int_{\mathbb{R}} \int_{0}^{+\infty} \tau^{\frac{1}{p}-i \xi_{1}-1} \varphi\left(\tau, y_{2}\right) d \tau e^{-i y_{2} \xi_{2}} d y_{2} d \xi_{1} e^{i \xi_{2} x_{2}} d \xi_{2}
\end{aligned}
$$

Hence, if we denote by $M_{1}$ the Mellin transform in the first variable of a sufficiently regular funciton $\psi$ defined on $(0,+\infty) \times \mathbb{R}$, that is,

$$
M_{1} \varphi\left(z, \xi_{2}\right)=\int_{0}^{+\infty} t^{z-1} \varphi\left(t, \xi_{2}\right) d t
$$

and by $\mathcal{F}_{2}$ the Fourier transform in the second variable, we see that

$$
\begin{aligned}
\mathcal{C}_{p}^{-1} T_{m_{p}} \mathcal{C}_{p}(\varphi)\left(t, x_{2}\right) & \\
= & \frac{1}{(2 \pi)^{2}} \int_{\mathbb{R}} \int_{\mathbb{R}} t^{-\frac{1}{p}+i \xi_{1}} m\left(\frac{1}{p}-i \xi_{1}, \xi_{2}\right)\left(M_{1} \mathcal{F}_{2} \varphi\right)\left(\frac{1}{p}-i \xi_{1}, \xi_{2}\right) d \xi_{1} e^{i \xi_{2} x_{2}} d \xi_{2} .
\end{aligned}
$$

Now, for every $\xi_{2}$ fixed, the function $M_{1}\left(\mathcal{F}_{2} \varphi\left(\cdot, \xi_{2}\right)\right)$ is holomorphic in the right half-plane and bounded in every closed strip $\overline{S_{\delta, R}}$, with $0<\delta<R<\infty$, and integrable on every vertical line in the right half-plane. Thus, for every $\xi_{2}$ fixed, $m\left(\cdot, \xi_{2}\right) M_{1}\left(\mathcal{F}_{2} \varphi\left(\cdot, \xi_{2}\right)\right)$ is holomorphic and bounded in every closed strip contained in $S_{a, b}$, and integrable on every vertical line in $S_{a, b}$. A 
standard application of Cauchy's theorem, as in the inversion of the Mellin transform, shows that

$$
\int_{c+i \mathbb{R}} t^{-z} m\left(z, \xi_{2}\right) M_{1}\left(\mathcal{F}_{2} \varphi\left(\cdot, \xi_{2}\right)\right)(z) d z
$$

is independent of $c \in(a, b)$. Thus, from (5) we have

$$
\mathcal{C}_{p}^{-1} T_{m_{p}} \mathcal{C}_{p}(\varphi)\left(t, x_{2}\right)=\frac{1}{(2 \pi)^{2}} \int_{\mathbb{R}}\left(\int_{\frac{1}{p}+i \mathbb{R}} t^{-z} m\left(z, \xi_{2}\right)\left(M_{1} \mathcal{F}_{2} \varphi\right)\left(z, \xi_{2}\right) d z\right) e^{i \xi_{2} x_{2}} d \xi_{2}
$$

and the conclusion follows.

Remark. Notice that we have shown that if $m$ satisfies the hypotheses of Thm. 2.1 then

$$
\mathcal{C}_{p}^{-1} T_{m_{p}} \mathcal{C}_{p}(\varphi)=\mathcal{F}_{2}^{-1} M_{1}^{-1}\left(m\left(M_{1} \mathcal{F}_{2} \varphi\right)\right)
$$

Equality ([6) clearly explains why we call the operator $\mathscr{T}_{m}$ a Mellin-Fourier multiplier operator. It equals a Mellin transformation in the first variable, a Fourier transformation in the second variable, followed by multiplication by $m$ and then the inverses of the Mellin and Fourier transforms.

Notice that, if $m, \tilde{m}$ satisfty the assumptions in Thm. 2.1, then $\mathscr{T}_{m} \mathscr{T}_{\tilde{m}}=\mathscr{T}_{m \tilde{m}}$.

\section{The Hardy SpaCe $\mathscr{H}^{p}\left(D_{\beta}\right)$}

In this section we show that the definition of $\mathscr{H}^{p}\left(D_{\beta}\right)$ is a very natural one, we exhibit an explicit formula for the Szegö projection $\mathscr{P}$ and we prove the sufficient condition in Theorem 1.

For every fixed $z_{2}$ such that $\left.|\log | z_{2}\right|^{2} \mid<\beta-\frac{\pi}{2}$ let us consider the half-plane

$$
\mathcal{U}_{z_{2}}:=\left\{z_{1}: \operatorname{Re}\left(z_{1} e^{-i \log \left|z_{2}\right|^{2}}\right)>0\right\} .
$$

In order to define $\mathscr{H}^{p}\left(D_{\beta}\right)$, it would be natural to consider a condition that would guarantee that, for each $z_{2}$ fixed, the function $f\left(\cdot, z_{2}\right)$ belongs to the Hardy space on $\mathcal{U}_{z_{2}}$. Indeed, this is the case for the space $\mathscr{H}^{p}\left(D_{\beta}\right)$.

Proposition 3.1. Let $1<p<\infty$ be given.

(i) Let $f \in \mathscr{H}^{p}\left(D_{\beta}\right)$. Then, for every fixed $z_{2}$ such that $\left.|\log | z_{2}\right|^{2} \mid<\beta-\frac{\pi}{2}$, the function $f_{z_{2}}:=f\left(\cdot, z_{2}\right)$ belongs to the (classical) Hardy space on the half-plane $\mathcal{U}_{z_{2}}, H^{p}\left(\mathcal{U}_{z_{2}}\right)$.

(ii) If $f \in \mathscr{H}^{p}\left(D_{\beta}\right)$, then $f$ admits boundary values on $\partial D_{\beta}$, that we still denote by $f$, and we have

$$
\|f\|_{\mathscr{H}^{p}\left(D_{\beta}\right)}=\|f\|_{L^{p}\left(d_{b}\left(D_{\beta}\right)\right)} .
$$

Proof. Suppose for the moment that $z_{2}$ is such that $\log \left|z_{2}\right|^{2}=-\frac{\pi}{2}$, so that $\mathcal{U}_{z_{2}}=\left\{z_{1}: \operatorname{Im} z_{1}>\right.$ $0\}$. We want to prove that $f_{z_{2}}$ satisfies

$$
\sup _{y>0} \int_{\mathbb{R}}\left|f_{z_{2}}(x+i y)\right|^{p} d x<\infty .
$$

From (3) it follows that $f_{z_{2}}$ satisfies

$$
\sup _{y \in\left(-\frac{\pi}{2}, \frac{\pi}{2}\right)} \int_{0}^{\infty}\left|f_{z_{2}}\left(r e^{i\left(t+\frac{\pi}{2}\right)}\right)\right|^{p} d r<\infty .
$$


In Sed75 is proved that conditions (7) and (8) actually define the same space on the upper half-plane. The same argument can be repeated for each $z_{2}$ such that $\left.|\log | z_{2}\right|^{2} \mid<\beta-\frac{\pi}{2}$ and conclusion (i) follows.

For the reader's convenience, we postpone the proof of (ii) to Section 6.

3.1. The Szegő projection $\mathscr{P}$. We now describe the operator $\mathscr{P}$ and show how it is related to the Mellin-Fourier multiplier operators studied in Section 2 ,

We first notice that

$$
d_{b}\left(D_{\beta}\right)=E^{+} \cup E^{-}
$$

where

$$
E^{+}=\left\{\left(\rho e^{i \beta}, e^{\frac{1}{2}\left(\beta-\frac{\pi}{2}\right)} e^{i \theta}\right) \in \mathbb{C}^{2}: \rho \in \mathbb{R}, \theta \in[0,2 \pi)\right\}
$$

and

$$
E^{-}=\left\{\left(\rho e^{-i \beta}, e^{-\frac{1}{2}\left(\beta-\frac{\pi}{2}\right)} e^{i \theta}\right) \in \mathbb{C}^{2}: \rho \in \mathbb{R}, \theta \in[0,2 \pi)\right\}
$$

and we remark that both $E^{+}$and $E^{-}$can be identified with $\mathbb{R} \times \mathbb{T}$. We write

$$
E^{+}=E_{0}^{+} \cup E_{1} \cup E_{2} \quad \text { and } \quad E^{-}=E_{0}^{-} \cup E_{3} \cup E_{4}
$$

where any component $E_{\ell}, \ell=1, \ldots, 4$, can be identified with $\mathbb{R}^{+} \times \mathbb{T}$ and $E_{0}^{+}, E_{0}^{-}$are sets of $d \sigma$-measure zero. Therefore, these latter sets can be disregarded on what follows. In detail, we have

$$
\begin{aligned}
& E_{1}=\left\{\left(\rho e^{i \beta}, e^{\frac{1}{2}\left(\beta-\frac{\pi}{2}\right)} e^{i \theta}\right): \rho \in \mathbb{R}^{+}, \theta \in[0,2 \pi)\right\} ; \\
& E_{2}=\left\{\left(-\rho e^{i \beta}, e^{\frac{1}{2}\left(\beta-\frac{\pi}{2}\right)} e^{i \theta}\right): \rho \in \mathbb{R}^{+}, \theta \in[0,2 \pi)\right\} \\
& E_{0}^{+}=\left\{\left(0, e^{\frac{1}{2}\left(\beta-\frac{\pi}{2}\right)} e^{i \theta}\right): \theta \in[0,2 \pi)\right\}
\end{aligned}
$$

and

$$
\begin{aligned}
& E_{3}=\left\{\left(\rho e^{-i \beta}, e^{-\frac{1}{2}\left(\beta-\frac{\pi}{2}\right)} e^{i \theta}\right): \rho \in \mathbb{R}^{+}, \theta \in[0,2 \pi)\right\} ; \\
& E_{4}=\left\{\left(-\rho e^{-i \beta}, e^{-\frac{1}{2}\left(\beta-\frac{\pi}{2}\right)} e^{i \theta}\right): \rho \in \mathbb{R}^{+}, \theta \in[0,2 \pi)\right\} ; \\
& E_{0}^{-}=\left\{\left(0, e^{-\frac{1}{2}\left(\beta-\frac{\pi}{2}\right)} e^{i \theta}\right): \theta \in[0,2 \pi)\right\} .
\end{aligned}
$$

Consequently, if $\chi_{\ell}$ denotes the characteristic function of $E_{\ell}$, by linearity, the operator $\mathscr{P}$ can be decomposed as

$$
\mathscr{P}=\sum_{k, \ell=1, \ldots, 4} \chi_{k} \mathscr{P} \chi_{\ell}:=\sum_{, \ell^{\prime}=1}^{4} \mathscr{P}_{k, \ell} .
$$

Therefore, we can study the continuity of $\mathscr{P}$ on $L^{p}\left(d_{b}\left(D_{\beta}\right)\right)$ and $W^{s, 2}\left(d_{b}\left(D_{\beta}\right)\right)$ by studying the continuity of the operators $\mathscr{P}_{k, \ell}$ on the same spaces.

It turns out that the operators $\mathscr{P}_{k, \ell}$ can be expressed in terms of the model operators $\mathscr{T}_{m}$ studied in Section 2, In order to show this, we set some notation.

Definition 3.2. For $z \in \mathbb{C}$ and $j \in \mathbb{Z}$ we set

$$
D(z, j)=4 \cosh \left[i \pi\left(z-\frac{1}{2}\right)\right] \cosh \left[i(2 \beta-\pi)\left(z-\frac{1}{2}+i\left(\frac{j}{2}+\frac{1}{4}\right)\right)\right]
$$


and

$$
S_{\nu_{\beta}}=\left\{z \in \mathbb{C}: \frac{1-\nu_{\beta}}{2}<z<\frac{1+\nu_{\beta}}{2}\right\} .
$$

Moreover, for $k, \ell \in\{1, \ldots, 4\}$ we set

$$
m_{k, \ell}(z, j)=\frac{e^{i \mu\left(z-\frac{1}{2}\right)} e^{i \eta\left(z-\frac{1}{2}+i \frac{j}{2}\right)}}{D(z, j)}
$$

where

$$
\begin{array}{lr}
\mu=-\pi \text { and } \eta=-(2 \beta-\pi) & \text { if }(k, \ell)=(1,1) ; \\
\mu=\pi \text { and } \eta=-(2 \beta-\pi) & \text { if }(k, \ell)=(2,2) ; \\
\mu=\pi \text { and } \eta=(2 \beta-\pi) & \text { if }(k, \ell)=(3,3) ; \\
\mu=-\pi \text { and } \eta=(2 \beta-\pi) & \text { if }(k, \ell)=(4,4) ; \\
\mu=0 \text { and } \eta=-(2 \beta-\pi) & \text { if }(k, \ell) \in\{(2,1),(1,2)\} ; \\
\mu=0 \text { and } \eta=(2 \beta-\pi) & \text { if }(k, \ell) \in\{(4,3),(3,4)\} ; \\
\mu=-\pi \text { and } \eta=0 & \text { if }(k, \ell) \in\{(4,1),(1,4)\} ; \\
\mu=\pi \text { and } \eta=0 & \text { if }(k, \ell) \in\{(3,2),(2,3)\} ; \\
\mu=0 \text { and } \eta=0 & \text { if }(k, \ell) \in\{(1,3),(3,1),(2,4),(4,2)\} .
\end{array}
$$

Then, the following theorem holds.

Theorem 3.3. For all $k, \ell \in\{1, \ldots, 4\}$ it holds that $\mathscr{P}_{k, \ell}=\mathscr{T}_{m_{k, \ell}}$, where the multipliers $m_{k, \ell}$ : $S_{\nu_{\beta}} \times \mathbb{Z}: \rightarrow \mathbb{C}$ are as in Def. (3.2). In particular, for $\frac{2}{1+\nu_{\beta}}<p<\frac{2}{1-\nu_{\beta}}$ the operator $\mathscr{P}_{k, \ell}$, $k, \ell \in\{1, \ldots, 4\}$, extend to bounded operator

$$
\mathscr{P}_{k, \ell}: L^{p}\left(d_{b}\left(D_{\beta}\right)\right) \rightarrow L^{p}\left(d_{b}\left(D_{\beta}\right)\right) .
$$

For the reader's convenience, we prove here only that the multipliers $m_{k, \ell}$ satisfy the hypothesis of Thm. 2.1. whereas we postpone to Section 6 the proof that the operators $\mathscr{P}_{k, \ell}$ actually are Mellin-Fourier multiplier operators.

Proof. It is immediate to verify that the multiplier $m_{k, \ell}$ satisfies hypothesis $(i)$ in Theorem 2.1 for every $k, \ell=1, \ldots, 4$. Concerning hypothesis $(i i)$, we proceed as follows. Let be $\sigma$ such that $\left(1-\nu_{\beta}\right) / 2<\sigma<\left(1+\nu_{\beta}\right) / 2$. Then, we want to prove that the function $m_{k, \ell}(\sigma+i \cdot, \cdot): \mathbb{R} \times \mathbb{Z} \rightarrow \mathbb{C}$ is a Fourier multiplier on $L^{p}(\mathbb{R} \times \mathbb{T})$. This is obtained by transference (see, e.g., Gra08, Chapter 3] from the fact that the extended function $m_{k, \ell}(\sigma+i \cdot, \cdot): \mathbb{R}^{2} \rightarrow \mathbb{C}$,

$$
m_{k, \ell}(\sigma+i \xi, \lambda)=\frac{e^{i \mu\left(\sigma-\frac{1}{2}+i \xi\right)} e^{i \eta\left(\sigma-\frac{1}{2}+i(\xi+\lambda)\right)}}{4 \cosh \left(i \pi\left(\sigma-\frac{1}{2}+i \xi\right)\right) \cosh \left(i(2 \beta-\pi)\left(\sigma-\frac{1}{2}+i\left(\xi+\lambda+\frac{1}{4}\right)\right)\right.}
$$

is a Fourier multiplier on $L^{p}\left(\mathbb{R}^{2}\right)$. This last affirmation is easily proved by showing that the composition of $m_{k, \ell}(\sigma+i \xi, \lambda)$ with the affine change of variables

$$
\left\{\begin{array}{l}
\xi^{\prime}=\pi \xi \\
\lambda^{\prime}=(2 \beta-\pi)(\xi+\lambda+1 / 4),
\end{array}\right.
$$


is a Mihlin-Hörmander multiplier (see, e.g, Gra08, Chapter 5]). This concludes the first part of the proof of Theorem 3.3 .

We observe that Theorem 3.3 immediately gives proves the positive results in Theorem 11. In the next section we prove the negative results for $\mathscr{P}$ in both $L^{p}$ and Sobolev scales.

\section{4. $L^{p}$ AND SobOlev IRREgularity}

In this section we provide an explicit counterexample to prove the negative result of Theorems 1 and 2 .

Let $\chi_{1}$ be the characteristic function of $E_{1} \subseteq d_{b}\left(D_{\beta}\right)$ defined in (9). Then, we define on $d_{b}\left(D_{\beta}\right)$ the function

$$
g\left(z_{1}, z_{2}\right):=\chi_{1}\left(z_{1}, z_{2}\right) e^{4 \beta\left(\beta+i \log \left|z_{1}\right|\right)} e^{-\left(\log \left|z_{1}\right|\right)^{2}-\frac{1}{2} \log \left|z_{1}\right|} .
$$

The following lemma is elementary, therefore we omit the proof.

Lemma 4.1. The function $g$ belongs to $L^{p}\left(d_{b}\left(D_{\beta}\right)\right)$ and $W^{s, p}\left(d_{b}\left(D_{\beta}\right)\right)$ for every $p \in(1, \infty)$ and positive integer $s$.

Next we focus on the Szegő projection of $g$. From (111) we deduce that $\mathscr{P} g=\sum_{\ell=1}^{4} \mathscr{P}_{\ell, 1} g$. We want to explicitly compute $\mathscr{P}_{1,1} g$, using Theorem 3.3 . We have that

$$
\mathcal{C}_{2} g\left(x e^{i \beta}, e^{\frac{1}{2}\left(\beta-\frac{\pi}{2}\right)} e^{i \theta}\right)=e^{4 \beta^{2}} e^{-x^{2}+i 4 \beta x},
$$

so that

$$
\begin{aligned}
\mathcal{F C}_{2} g(\xi, j) & =\mathcal{F}_{1} \mathcal{C}_{2} g(\xi) \\
& =e^{4 \beta^{2}} \int_{\mathbb{R}} e^{i 4 \beta x} e^{-x^{2}} e^{-i x \xi} d x \\
& =C_{\beta} e^{-\frac{(\xi-4 \beta)^{2}}{4}},
\end{aligned}
$$

where $C_{\beta}$ is a positive constant depending on $\beta$. In conclusion, we obtain that, up to a multiplicative positive constant,

$$
\mathscr{P}_{1,1} g(x):=\mathscr{P}_{1,1} g\left(x e^{i \beta}, e^{\frac{1}{2}\left(\beta-\frac{\pi}{2}\right)} e^{i \theta}\right)=\mathcal{C}_{2}^{-1} \mathcal{F}^{-1}\left[\frac{e^{-\frac{(\cdot)^{2}}{4}}}{\cosh (\pi \cdot) \cosh ((2 \beta-\pi) \cdot)}\right](x) .
$$

We recall once again that, by definition, $\mathscr{P}_{1,1} g$ is supported on $E_{1}$; hence $x$ is always assumed to be positive.

If we define the functions

$$
f(\xi)=e^{-\frac{\xi^{2}}{4}} ; \quad h(\xi)=\frac{1}{\cosh (\pi \xi)} ; \quad r(\xi)=\frac{1}{\cosh ((2 \beta-\pi) \xi)},
$$

then, it is easy to prove that,

$$
\mathcal{F}^{-1} f(x)=\frac{e^{-x^{2}}}{\sqrt{\pi}} ; \quad \mathcal{F}^{-1} h(x)=\frac{1}{2 \pi} \frac{1}{\cosh \left(\frac{x}{2}\right)} ; \quad \mathcal{F}^{-1} r(x)=\frac{\nu_{\beta}}{2 \pi} \frac{1}{\cosh \left(\frac{\nu_{\beta} x}{2}\right)}
$$

where $\nu_{\beta}=\frac{\pi}{2 \beta-\pi}$. 
Hence,

$$
\mathcal{F}^{-1}\left[\frac{e^{-\frac{(\cdot)^{2}}{4}}}{\cosh (\pi \cdot) \cosh ((2 \beta-\pi) \cdot)}\right](x)=C_{\beta} \int_{\mathbb{R}} \int_{\mathbb{R}} \frac{e^{-s^{2}}}{\cosh \left(\frac{\nu_{\beta}(x-t)}{2}\right) \cosh \left(\frac{t-s}{2}\right)} d s d t .
$$

We now prove two lemmas.

Lemma 4.2. There exist two positive constants $A$ and $B$ such that

$$
A x^{-\frac{\nu_{\beta}+1}{2}} \leq \mathscr{P}_{1,1} g(x) \leq B x^{-\frac{\nu_{\beta}+1}{2}}
$$

for every $x \in(1, \infty)$.

Proof. From (14) and (15) we get

$$
\begin{aligned}
x^{\frac{1+\nu_{\beta}}{2}} \mathscr{P}_{1,1} g(x) & =C_{\beta} x^{\frac{\nu_{\beta}}{2}} \int_{\mathbb{R}} \int_{\mathbb{R}} \frac{1}{\cosh \left(\frac{\nu_{\beta}(\log x-t)}{2}\right)} \frac{e^{-s^{2}}}{\cosh \left(\frac{t-s}{2}\right)} d s d t \\
& =C_{\beta} \int_{\mathbb{R}} \int_{\mathbb{R}} \frac{2}{e^{-\frac{\nu_{\beta} t}{2}}+e^{-\nu_{\beta} \log x} e^{\frac{\nu_{\beta} t}{2}}} \frac{e^{-s^{2}}}{\cosh \left(\frac{t-s}{2}\right)} d s d t .
\end{aligned}
$$

Now, since $x>1$,

$$
\begin{aligned}
& C_{\beta} \int_{\mathbb{R}} \int_{\mathbb{R}} \frac{2}{e^{-\frac{\nu_{\beta} t}{2}}+e^{-\nu_{\beta} \log x} e^{\frac{\nu_{\beta} t}{2}}} \frac{e^{-s^{2}}}{\cosh \left(\frac{t-s}{2}\right)} d s d t \geq C_{\beta} \int_{\mathbb{R}} \int_{\mathbb{R}} \frac{1}{\cosh \left(\frac{\nu_{\beta} t}{2}\right)} \frac{e^{-s^{2}}}{\cosh \left(\frac{t-s}{2}\right)} d s d t \\
& =: \text { A. }
\end{aligned}
$$

Similarly,

$$
\begin{aligned}
C_{\beta} \int_{\mathbb{R}} \int_{\mathbb{R}} \frac{2}{e^{-\frac{\nu_{\beta} t}{2}}+e^{-\nu_{\beta} \log x} e^{\frac{\nu_{\beta} t}{2}}} \frac{e^{-s^{2}}}{\cosh \left(\frac{t-s}{2}\right)} d s d t & \leq C_{\beta} \int_{\mathbb{R}} \int_{\mathbb{R}} \frac{2}{e^{-\frac{\nu_{\beta} t}{2}}} \frac{e^{-s^{2}}}{\cosh \left(\frac{t-s}{2}\right)} d s d t \\
& =: B .
\end{aligned}
$$

Thus, the lemma is proved.

Lemma 4.3. There exist two positive constants $A$ and $B$ such that

$$
A x^{\frac{\nu_{\beta}-1}{2}} \leq \mathscr{P}_{1,1} g(x) \leq B x^{\frac{\nu_{\beta}-1}{2}}
$$

for every $x \in(0,1)$.

Proof. From (14) and (15) we obtain

$$
x^{\frac{1-\nu_{\beta}}{2}} \mathscr{P}_{1,1} g(x)=C_{\beta} \int_{\mathbb{R}} \int_{\mathbb{R}} \frac{2}{e^{\nu_{\beta} \log x} e^{-\frac{\nu_{\beta} t}{2}}+e^{\frac{\nu_{\beta} t}{2}}} \frac{e^{-s^{2}}}{\cosh \left(\frac{t-s}{2}\right)} d s d t .
$$

Now, since $x \in(0,1)$,

$$
\begin{aligned}
C_{\beta} \int_{\mathbb{R}} \int_{\mathbb{R}} \frac{2}{e^{\nu_{\beta} \log x} e^{-\frac{\nu_{\beta} t}{2}}+e^{\frac{\nu_{\beta} t}{2}}} \frac{e^{-s^{2}}}{\cosh \left(\frac{t-s}{2}\right)} d s d t & \geq C_{\beta} \int_{\mathbb{R}} \int_{\mathbb{R}} \frac{1}{\cosh \left(\frac{\nu_{\beta} t}{2}\right)} \frac{e^{-s^{2}}}{\cosh \left(\frac{t-s}{2}\right)} d s d t \\
& =: A .
\end{aligned}
$$


Similarly,

$$
\begin{aligned}
C_{\beta} \int_{\mathbb{R}} \int_{\mathbb{R}} \frac{2}{e^{\nu_{\beta} \log x} e^{-\frac{\nu_{\beta} t}{2}}+e^{\frac{\nu_{\beta} t}{2}}} \frac{e^{-s^{2}}}{\cosh \left(\frac{t-s}{2}\right)} d s d t & \leq C_{\beta} \int_{\mathbb{R}} \int_{\mathbb{R}} \frac{2}{e^{\frac{\nu_{\beta} t}{2}}} \frac{e^{-s^{2}}}{\cosh \left(\frac{t-s}{2}\right)} d s d t \\
=: B & =: B .
\end{aligned}
$$

and the proof is concluded.

We point out that the constants $A$ and $B$ in Lemma 4.2 and Lemma 4.3 are the same.

The necessary condition in Theorem 1 is immediately deduced.

Necessary condition of Theorem 1. It follows easily from Lemmas 4.2 and 4.3 that $\mathscr{P}_{1,1} g$ is not in $L^{p}$ unless $\frac{2}{1+\nu_{\beta}}<p<\frac{2}{1-\nu_{\beta}}$. Hence, it follows from Lemma 4.1 that the operator $\mathscr{P}$ cannot be bounded on $L^{p}$ unless $\frac{2}{1+\nu_{\beta}}<p<\frac{2}{1-\nu_{\beta}}$ and the proof is concluded.

4.1. The $W^{s, 2}$ irregularity. As mentioned in the introduction, the fractional Sobolev spaces we consider are the ones defined via Fourier transform and Bessel potentials. Namely, the space $W^{s, p}\left(b_{d}\left(D_{\beta}\right)\right)$ is defined by the norm

$$
\|f\|_{W^{s, p}\left(d_{b}\left(D_{\beta}\right)\right)}^{p}=\sum_{\ell=1}^{4}\|f\|_{W^{s, p}\left(E_{\ell}\right)}^{p}
$$

where

$$
\|f\|_{W^{s, p}\left(E_{\ell}\right)}^{p}=\|f\|_{W^{s, p}(\mathbb{R} \times \mathbb{T})}^{p}=\int_{\mathbb{R} \times \mathbb{T}}\left|\mathcal{F}^{-1}\left[\left[1+(\cdot)^{2}+(\cdot)^{2}\right]^{\frac{s}{2}} \mathcal{F} f(\cdot, \cdot)\right](x, \theta)\right|^{p} d x d \theta .
$$

We point out that in the definition of the Sobolev norm we are integrating with respect to the Lebesgue measure $d x d \theta$ and not with respect to $d \sigma$, the induced measure on $E_{\ell}$. It is easy to verify that, up to a multiplicative positive constant, these two measure coincides.

Obviously, a different definition of fractional Sobolev spaces can be used, but, due to the nature of the operator $\mathscr{P}$, this definition is the most natural one for our setting.

We now focus on the Hilbert space $W^{s, 2}\left(d_{b}\left(D_{\beta}\right)\right)$. In particular, we want to estimate the $W^{s, 2}$ of $\mathscr{P} g$ where $g$ is the function (13) once again. Notice that, since the function $g$ does not depend on the periodic variable on its support, it holds

$$
\|g\|_{W^{s, 2}(\mathbb{R} \times \mathbb{T})}^{2}=\int_{\mathbb{R}}\left|\mathcal{F}^{-1}\left[\left[1+(\cdot)^{2}\right]^{\frac{s}{2}} \mathcal{F} g(\cdot)\right](x)\right|^{2} d x=\|g\|_{W^{s, 2}(\mathbb{R})}^{2},
$$

An equivalent norm on $W^{s, 2}(\mathbb{R})$ is given by

$$
\|g\|_{s, 2}^{2}=\|g\|_{W[s], 2}^{2}+[g]_{s, 2}
$$

where $[s]$ denotes the integer part of $s,\|g\|_{W^{[s], 2}}$ is the classical Sobolev norm of integer order $[s]$ and $[g]_{s, 2}$ is the so-called Gagliardo seminorm, that is,

$$
[g]_{s, 2}=\int_{\mathbb{R}} \int_{\mathbb{R}} \frac{\left|D^{[s]} g(x)-D^{[s]} g(y)\right|^{2}}{|x-y|^{1+2 s}} d x d y .
$$

For the equivalence between the norms (16) and (17) we refer the reader to [Tri83] where the spaces identified by these two norms are seen as special cases of the more general Triebel spaces.

We are ready to prove our result. 
Necessary condition of Theorem Q 2 . Let us assume for the moment that $s \in(0,1)$. Because of (11) it holds

$$
\int_{d_{b}\left(D_{\beta}\right)} \int_{d_{b}\left(D_{\beta}\right)} \frac{|\mathscr{P} g(x)-\mathscr{P} g(y)|^{2}}{|x-y|^{1+2 s}} d x d y \geq \int_{0}^{\infty} \int_{0}^{\infty} \frac{\left|\mathscr{P}_{1,1} g(x)-\mathscr{P}_{1,1} g(y)\right|^{2}}{|x-y|^{1+2 s}} d x d y .
$$

Suppose now that $x$ and $y$ satisfies $1<y<\alpha x$ where $\alpha$ is a positive number to be fixed. Then, it follows from Lemma 4.2 that

$$
\begin{aligned}
\left|\mathscr{P}_{1,1} g(x)-\mathscr{P}_{1,1} g(y)\right| & \geq \mathscr{P}_{1,1} g(y)-\mathscr{P}_{1,1} g(x) \\
& \geq A y^{-\frac{1+\nu_{\beta}}{2}}-B x^{-\frac{1+\nu_{\beta}}{2}} \\
& \geq A y^{-\frac{1+\nu_{\beta}}{2}}-B\left(\frac{y}{\alpha}\right)^{-\frac{1+\nu_{\beta}}{2}} \\
& =y^{-\frac{1+\nu_{\beta}}{2}}\left[A-\alpha^{\frac{1+\nu_{\beta}}{2}} B\right] \\
& \geq \frac{A}{2} y^{-\frac{1+\nu_{\beta}}{2}}
\end{aligned}
$$

if $\alpha$ is chosen such that $\alpha<\left(\frac{A}{2 B}\right)^{\frac{2}{1+\nu_{\beta}}}$. In particular, this implies that $\alpha<\frac{1}{2}$ since $B>A$ and $\frac{1+\nu_{\beta}}{2}<1$. Therefore,

$$
\begin{aligned}
\int_{0}^{\infty} \int_{0}^{\infty} \frac{\left|\mathscr{P}_{1,1} g(x)-\mathscr{P}_{1,1} g(y)\right|^{2}}{|x-y|^{1+2 s}} & d x d y \geq \int_{\{1<y<\alpha x\}} \frac{\left|\mathscr{P}_{1,1} g(x)-\mathscr{P}_{1,1} g(y)\right|^{2}}{|x-y|^{1+2 s}} d x d y \\
& =\int_{0}^{\infty} \int_{0}^{\arctan \alpha} \frac{\left|\mathscr{P}_{1,1} g\left(\frac{1}{\alpha}+\rho \cos \theta\right)-\mathscr{P}_{1,1} g(1+\rho \sin \theta)\right|^{2}}{\left|\frac{1}{\alpha}+\rho \cos \theta-1-\rho \sin \theta\right|^{1+2 s}} \rho d \theta d \rho \\
& \geq \frac{A^{2}}{4} \int_{0}^{\infty} \int_{0}^{\arctan \alpha} \frac{\left|(1+\rho \sin \theta)^{-\frac{1+\nu_{\beta}}{2}}\right|^{2}}{\left|\frac{1}{\alpha}-1+\rho(\cos \theta-\sin \theta)\right|^{1+2 s}} \rho d \theta d \rho .
\end{aligned}
$$

Notice that the integration in $d \theta$ of this last integral is finite for every $\rho>0$ since $\alpha<\frac{1}{2}$. Thus, we only have to discuss the integration in $d \rho$. It holds,

$$
\begin{aligned}
\int_{0}^{\infty} \int_{0}^{\infty} \frac{\left|\mathscr{P}_{1,1} g(x)-\mathscr{P}_{1,1} g(y)\right|^{2}}{|x-y|^{1+2 s}} d x d y & \geq \frac{A^{2}}{4} \int_{0}^{\infty} \int_{0}^{\arctan \alpha} \frac{\left|(1+\rho \sin \theta)^{-\frac{1+\nu_{\beta}}{2}}\right|^{2}}{\left|\frac{1}{\alpha}-1+\rho(\cos \theta-\sin \theta)\right|^{1+2 s}} \rho d \theta d \rho \\
& \geq \frac{A^{2}}{4} \int_{0}^{\infty} \int_{0}^{\arctan \alpha} \frac{\left|(1+\rho)^{-\frac{1+\nu_{\beta}}{2}}\right|^{2}}{\left|\frac{1}{\alpha}-1+2 \rho\right|^{1+2 s}} \rho d \theta d \rho .
\end{aligned}
$$

This integral is finite if and only if $s>-\frac{\nu_{\beta}}{2}$ and this condition is trivially satisfied since we are assuming $s$ to be positive.

Suppose now that $x$ and $y$ satisfies $1>y>\frac{x}{\alpha}$. Then, from Lemma 4.3, we obtain

$$
\begin{aligned}
\left|\mathscr{P}_{1,1} g(x)-\mathscr{P}_{1,1} g(y)\right| & \geq \mathscr{P}_{1,1} g(y)-\mathscr{P}_{1,1} g(x) \\
& \geq A y^{\frac{\nu_{\beta}-1}{2}}-B x^{\frac{\nu_{\beta}-1}{2}} \\
& \geq y^{\frac{\nu_{\beta}-1}{2}}\left[A-\alpha^{\frac{\nu_{\beta}-1}{2}} B\right] \\
& \geq \frac{A}{2} y^{\frac{\nu_{\beta}-1}{2}}
\end{aligned}
$$


if $\alpha$ chosen that $\frac{1}{\alpha}<\left(\frac{A}{2 B}\right)^{\frac{2}{1-\nu_{\beta}}}$. Since $B>A$, it follows $\alpha>2$. Therefore,

$$
\begin{aligned}
\int_{0}^{\infty} \int_{0}^{\infty} \frac{\left|\mathscr{P}_{1,1} g(x)-\mathscr{P}_{1,1} g(y)\right|^{2}}{|x-y|^{1+2 s}} d x d y & \geq \int_{\left\{\frac{x}{\alpha}<y<1\right\}} \frac{\left|\mathscr{P}_{1,1} g(x)-\mathscr{P}_{1,1} g(y)\right|^{2}}{|x-y|^{1+2 s}} d x d y \\
& \geq \int_{0}^{\frac{1}{2}} \int_{\arctan \frac{1}{\alpha}}^{\frac{\pi}{2}} \frac{\left|\mathscr{P}_{1,1} g(\rho \cos \theta)-\mathscr{P}_{1,1} g(\rho \sin \theta)\right|^{2}}{\rho^{1+2 s}|\cos \theta-\sin \theta|} \rho d \theta d \rho \\
& \geq \frac{A^{2}}{4} \int_{0}^{\frac{1}{2}} \int_{\arctan \frac{1}{\alpha}}^{\frac{\pi}{2}} \frac{\rho^{\nu_{\beta}-1}(\sin \theta)^{\nu_{\beta}-1}}{\rho^{s p}|\cos \theta-\sin \theta|^{1+s p}} d \theta d \rho .
\end{aligned}
$$

Notice that the integration in $d \theta$ is finite for every $\rho>0$ since $\frac{1}{\alpha}>2$. Instead, the integration in $d \rho$ is finite if and only if $s<\frac{\nu_{\beta}}{2}$.

Thus, we showed that for $s \in(0,1)$ the Gagliardo seminorm of $\mathscr{P} g$ is finite if and only if $0 \leq s<\frac{\nu_{\beta}}{2}$. Hence, because of the equivalence between (16) and (17), it holds that $\mathscr{P} g$ is in $W^{s, 2}(\mathbb{R} \times \mathbb{T})$ if and only if $0 \leq s<\frac{\nu_{\beta}}{2}$. This fact, together with Lemma 4.1, proves the necessary condition of Theorem 2 in the case $s \in(0,1)$.

Suppose now that $s \geq 1$. If $\mathscr{P}$ would be bounded on $W^{s, 2}(\mathbb{R} \times \mathbb{T})$, then it would be bounded on $W^{s_{1}, 2}(\mathbb{R} \times \mathbb{T})$ as well for every $s_{1} \in[0, s]$ by interpolation between $L^{2}$ and $W^{s, 2}$. This is a contradiction. Hence, we obtain that $\mathscr{P}$ is an unbounded operator on $W^{s, 2}(\mathbb{R} \times \mathbb{T})$ for every $s \geq \frac{\nu_{\beta}}{2}$ and the proof is concluded.

4.2. The $W^{s, p}$ irregularity. By the very same argument we used to prove the $W^{s, 2}$ irregularity $\mathscr{P}$ we prove Theorem 3 .

Unlike in the Hilbert setting, we do not have on $W^{s, p}(\mathbb{R})$ an equivalent norm in terms of Gagliardo seminorm similarly to (17). Nonetheless, there are results which link Bessel potential spaces with the Gagliardo seminorm.

For $s>0$ and $p \in(1, \infty)$ define the function space

$$
\mathcal{W}^{s, p}(\mathbb{R})=\left\{f \in \mathcal{W}^{[s], p}(\mathbb{R}):[f]_{s, p}:=\int_{\mathbb{R}} \int_{\mathbb{R}} \frac{\left|D^{[s]} f(x)-D^{[s]} f(y)\right|^{p}}{|x-y|^{1+s p}}<\infty\right\},
$$

where $[s]$ denotes the integer part of $s, D^{[s]} f$ is the $[s]$-derivative of the function $f$ and $\mathcal{W}^{[s], p}(\mathbb{R})$ is the classical Sobolev space of integer order $[s]$. The space $\mathcal{W}^{s, p}(\mathbb{R})$ is endowed with the norm

$$
\|f\|_{\mathcal{W}^{s, p}(\mathbb{R})}^{p}=\|f\|_{\mathcal{W}[s] \cdot p(\mathbb{R})}^{p}+[f]_{s, p} .
$$

As in the Hilbert setting, the spaces $W^{s, p}(\mathbb{R})$ and $\mathcal{W}^{s, p}(\mathbb{R})$ can be seen as special cases of Triebel spaces [Tri83].

As we mentioned, unless $p=2$, the spaces $W^{s, p}(\mathbb{R})$ and $\mathcal{W}^{s, p}(\mathbb{R})$ do not coincide; nonetheless, the following proposition holds.

Proposition 4.4. Let be $s>0$ and $p \in(1, \infty)$. Then, for every $\varepsilon>0$,

$$
W^{s+\varepsilon, p}(\mathbb{R}) \subseteq \mathcal{W}^{s, p}(\mathbb{R}) \subseteq W^{s-\varepsilon, p}(\mathbb{R}),
$$

where $A \subseteq B$ denotes continuous inclusion.

Moreover, if $p>2$, it holds

$$
W^{s, p}(\mathbb{R}) \subseteq \mathcal{W}^{s, p}(\mathbb{R}) .
$$

Proof. See Tri83]. 
Exploiting this last proposition we prove Theorem 3 ,

Proof of Theorem 13. Computing the Gagliardo seminorm of $\mathscr{P} g$ for $s \in(0,1)$ as in the Hilbert setting, we obtain that $\|\mathscr{P} g\|_{\mathcal{W}^{s, p}(\mathbb{R})}<\infty$ if and only if the stronger condition

$$
-\frac{\nu_{\beta}}{2}<s+\frac{1}{2}-\frac{1}{p}<\frac{\nu_{\beta}}{2}
$$

holds. Then, using (18) we conclude the proof in the case $s \in(0,1)$. If $s \geq 1$ we conclude using interpolation as in the Hilbert setting. In we assume also $p \geq 2$ we repeat the same argument using the stronger inclusion (19).

\section{Sobolev Regularity}

In this section we prove the positive result in Theorem 2, As in the $L^{p}$ setting, we deduce the boundedness of $\mathscr{P}$ from the boundedness of the operators $\mathscr{P}_{k, \ell}, k, \ell=1, \ldots, 4$.

To prove our result, we obtain an integral representation for the general operator $\mathscr{P}_{k, \ell}$ and then we exploit some classical results for Calderón-Zygmund singular integral operators Duo01, Gra08, Gra09.

From Theorem 3.3 we get

$$
\mathscr{P}_{k, \ell}=\chi_{k}\left(\mathcal{C}_{2}^{-1} T_{m_{\mu}} \mathcal{C}_{2}\right) \circ\left(\mathcal{C}_{2}^{-1} T_{m_{\eta}} \mathcal{C}_{2}\right) \chi_{\ell}
$$

where $T_{m_{\mu}}$ and $T_{m_{\eta}}$ are Fourier multiplier operators on $L^{2}(\mathbb{R} \times \mathbb{T})$ associated to the multipliers

$$
m_{\mu}(\xi)=\frac{e^{\mu \xi}}{2 \cosh (\pi \xi)} \quad \text { and } \quad m_{\eta}(\xi, j)=\frac{e^{\eta\left(\xi-\frac{j}{2}\right)}}{2 \cosh \left((2 \beta-\pi)\left(\xi-\frac{j}{2}-\frac{1}{4}\right)\right.}
$$

with $|\mu|=\pi$ or $\mu=0$ and $|\eta|=2 \beta-\pi$ or $\eta=0$ according to the scheme in Definition 3.2

Thus, we deduce the boundedness $\mathscr{P}_{k, \ell}: W^{s, 2}\left(d_{b}\left(D_{\beta}\right)\right) \rightarrow W^{s, 2}\left(d_{b}\left(D_{\beta}\right)\right)$, from the boundedness of the operators

$$
\begin{aligned}
P_{a} & =\chi_{+}\left(\mathcal{C}_{2}^{-1} T_{m_{a}} \mathcal{C}_{2}\right) \chi_{+} \\
Q_{a} & =\chi_{+}\left(\mathcal{C}_{2}^{-1} T_{M_{a}} \mathcal{C}_{2}\right) \chi_{+}
\end{aligned}
$$

where $\chi_{+}$denotes the characteristic function of $(0, \infty)$ and, for $|a| \geq \pi$,

$$
\begin{aligned}
m_{a}(\xi) & =\frac{e^{a \xi}}{2 \cosh (a \xi)}, \\
M_{a}(\xi, j) & =e^{\frac{a}{4}} m_{a}\left(\xi-\frac{j}{2}-\frac{1}{4}\right) .
\end{aligned}
$$

Remark 5.1. It should be noticed that the operators $P_{a}$ and $Q_{a}$ involve the multiplication by the characteristic function $\chi_{+}$, that obviously destroys some smoothness. However, we only need to consider the case of Sobolev spaces $W^{s, 2}$, with $0<s<\frac{\nu_{\beta}}{2}<\frac{1}{2}$ and the multiplication by $\chi_{+}$preserves the Sobolev spaces for such small values of $s$, as we will see.

We also point out that the multipliers $m_{a}$ and $M_{a}$ are not models for $m_{\mu}$ and $m_{\eta}$ respectively when $\mu=\eta=0$. Nonetheless, these cases are the easiest to deal with and the techniques we use can be easily adapted to these situations. 
Theorem 5.2. The operator $P_{a}$ defines a bounded operator

$$
P_{a}: W^{s, 2}(\mathbb{R} \times \mathbb{T}) \rightarrow W^{s, 2}(\mathbb{R} \times \mathbb{T})
$$

for every $s \in\left[0, \frac{\pi}{2|a|}\right)$.

In order to prove the theorem, we need a few preliminary results.

Lemma 5.3. Let be $|a| \geq \pi$ and $\kappa \in\left[0, \frac{\pi}{2|a|}\right)$ and consider the tempered distribution $U_{a, \kappa}$ defined by, for $g$ in the Schwartz space $\mathcal{S}(\mathbb{R})$,

$$
\left\langle U_{a, \kappa}, g\right\rangle=\lim _{\varepsilon \rightarrow 0} \int_{\varepsilon<\left|\frac{\pi \xi}{2 a}\right|} \frac{e^{-\kappa t}}{\sinh \left(\frac{\pi t}{2 a}\right)} g(t) d t .
$$

Then, the Fourier transform $\widehat{U_{a, \kappa}}$ is given by

$$
\widehat{U_{a, \kappa}}(\xi)=2|a| i \tanh (a(\xi-i \kappa)) .
$$

Proof. Writing

$$
\int_{\varepsilon<\left|\frac{\pi t}{2 a}\right|} \frac{e^{-\kappa t}}{\sinh \left(\frac{\pi t}{2 a}\right)} g(t) d t=\int_{\varepsilon<\left|\frac{\pi t}{2 a}\right| \leq 1} \frac{e^{-\kappa t} g(t)-g(0)}{\sinh \left(\frac{\pi t}{2 a}\right)} d t+\int_{1<\left|\frac{\pi t}{2 a}\right|} \frac{e^{-\kappa t}}{\sinh \left(\frac{\pi t}{2 a}\right)} g(t) d t
$$

it is easy to check that $U_{a, \kappa}$ is in fact a well-defined tempered distribution.

Next, a standard contour integration argument now shows that, for $\zeta \in \mathbb{C}$ with $|\operatorname{Im} \zeta|<1$,

$$
\int_{\varepsilon<|t|<R} \frac{e^{-i \zeta t}}{\sinh t} d t=i \pi \tanh \left(\frac{\pi}{2} \zeta\right)+o(1)
$$

as $\varepsilon \rightarrow 0^{+}$and $R \rightarrow+\infty$, so that

$$
\int_{\varepsilon<\left|\frac{\pi \xi}{2 a}\right|<R} \frac{e^{-i \zeta t}}{\sinh \left(\frac{\pi t}{2 a}\right)} d t=2|a| i \tanh (a \zeta)+o(1),
$$

as $\varepsilon \rightarrow 0^{+}$and $R \rightarrow+\infty$. Therefore, setting $\zeta=\xi-i \kappa$ we obtain (21) with $0 \leq \kappa<\frac{\pi}{2|a|}$.

Corollary 5.4. The following facts hold.

(i) The convolution operator $T_{a, \kappa}: g \mapsto U_{a, \kappa} * g$, densely defined on $\mathcal{S}(\mathbb{R})$, extends to a bounded operator $L^{2} \rightarrow L^{2}$ for every $\kappa \in\left[0, \frac{\pi}{2|a|}\right)$.

(ii) The Fourier multiplier operator $T_{m_{a}}$ is given by

$$
T_{m_{a}}=\frac{1}{2}\left(I+\frac{1}{2|a| i} T_{a, 0}\right) .
$$

(iii) The operator $T_{a, 0}$ is a Calderón-Zygmund singular integral operator. Therefore, for every $0<\varepsilon<R<\infty$, the truncated operator

$$
T_{a, 0,(\varepsilon, R)} g(x):=\int_{\varepsilon<\left|\frac{\pi t}{2 a}\right|<R} \frac{g(x-t)}{\sinh \left(\frac{\pi t}{2 a}\right)} d t
$$

densely defined on $\mathcal{S}(\mathbb{R})$ extends to a bounded operator $L^{2} \rightarrow L^{2}$ with operator norm independent of $\varepsilon$ and $R$. Moreover,

$$
\lim _{\substack{\varepsilon \rightarrow 0^{+} \\ R \rightarrow+\infty}} T_{a, 0,(\varepsilon, R)} g=T_{a, 0} g
$$

in $L^{2}(\mathbb{R})$. 
Proof. $(i)$ is obvious, since $\widehat{U_{a, \kappa}} \in L^{\infty}(\mathbb{R})$ for $\kappa \in\left[0, \frac{\pi}{2|a|}\right)$. Since

$$
\widehat{U_{a, 0}}(\xi)=2|a| i \tanh (a \xi)=2|a| i\left(\frac{e^{a \xi}}{\cosh (a \xi)}-1\right)=4|a| i\left(m_{a}(\xi)-\frac{1}{2}\right),
$$

(ii) also follows. Part (iii) follows from the standard theory of Calderón-Zygmund singular integral operators and we refer the reader, for instance, to [Duo01, Chapter 5].

From the previous corollary, we finally obtain an integral representation for the operator $P_{a}$. For $|a| \geq \pi$, we now set

$$
\Lambda_{a,(\varepsilon, R)}:=\chi_{+} \mathcal{C}_{2}^{-1} T_{a, 0,(\varepsilon, R)} \mathcal{C}_{2} \chi_{+}
$$

Corollary 5.5. The following facts hold.

(i) there exists a constant $C>0$, independent of $\varepsilon, R>0$ such that

$$
\left\|\Lambda_{a,(\varepsilon, R)} f\right\|_{L^{2}(\mathbb{R} \times \mathbb{T})} \leq C\|f\|_{L^{2}(\mathbb{R} \times \mathbb{T})}
$$

(ii) $\lim _{\substack{\varepsilon \rightarrow 0^{+} \\ R \rightarrow+\infty}} \Lambda_{a,(\varepsilon, R)} f:=\Lambda_{a} f$ exists in $L^{2}(\mathbb{R} \times \mathbb{T})$-norm, for every test function $f$, and it defines a bounded operator $\Lambda_{a}: L^{2}(\mathbb{R} \times \mathbb{T}) \rightarrow L^{2}(\mathbb{R} \times \mathbb{T})$;

(iii) For every $f \in L^{2}(\mathbb{R} \times \mathbb{T})$ the operator $P_{a}$ is given by

$$
\begin{aligned}
P_{a} f & =\frac{1}{2}\left(\chi_{+} f\right)+\frac{1}{2|a| i} \chi_{+}\left(\mathcal{C}_{2}^{-1} T_{a, 0} \mathcal{C}_{2}\right)\left(\chi_{+} f\right) \\
& =: \frac{1}{2} \Lambda_{+} f+\frac{1}{2|a| i} \Lambda_{a} f
\end{aligned}
$$

(iv) for $(\xi, j) \in \mathbb{R} \times \mathbb{Z}$ and $f \in L^{2}(\mathbb{R} \times \mathbb{T})$,

$$
\mathcal{F}\left(\Lambda_{a} f\right)(\xi, j)= \begin{cases}\Lambda_{a}\left(\mathcal{F}\left(\chi_{+} f\right)\right)(\xi, j) & \text { if } \xi>0 \\ \left.\Lambda_{a}\left(\widetilde{\mathcal{F}\left(\chi_{+} f\right.}\right)\right)(-\xi, j) & \text { if } \xi<0,\end{cases}
$$

where $\left.\left.\left.\widetilde{\mathcal{F}\left(\chi_{+} f\right.}\right)\right)(x, \theta):=\mathcal{F}\left(\chi_{+} f\right)\right)(-x, \theta)$.

Proof. From (iii) of Corollary 5.4 and recalling that $\mathcal{C}_{2}: L^{2}((0, \infty), \times \mathbb{T}) \rightarrow L^{2}(\mathbb{R} \times \mathbb{T})$ is an isometry, we get

$$
\begin{aligned}
\int_{\mathbb{T}} \int_{\mathbb{R}}\left|\Lambda_{a,(\varepsilon, R)} f(x, \theta)\right|^{2} d x d \theta & =\int_{\mathbb{T}} \int_{0}^{+\infty}\left|\mathcal{C}_{2}^{-1} T_{a, 0,(\varepsilon, R)} \mathcal{C}_{2}\left(\chi_{+} f\right)(x, \theta)\right|^{2} d x d \theta \\
& =\int_{\mathbb{T}} \int_{\mathbb{R}}\left|T_{a, 0,(\varepsilon, R)} \mathcal{C}_{2}\left(\chi_{+} f\right)(x, \theta)\right|^{2} d x d \theta \\
& \leq C \int_{\mathbb{T}} \int_{\mathbb{R}}\left|\mathcal{C}_{2}\left(\chi_{+} f\right)(x, \theta)\right|^{2} d x d \theta \\
& =C \int_{\mathbb{T}} \int_{0}^{+\infty}\left|\chi_{+}(x) f(x, \theta)\right|^{2} d x d \theta \\
& \leq C \int_{\mathbb{T}} \int_{\mathbb{R}}|f(x, \theta)|^{2} d x d \theta
\end{aligned}
$$

as we wished. Thus, $(i)$ is proved. With a similar argument, $(i i)$ is also proved. For $(i i i)$ the proof is easily deduced from these results, $(i i)$ of Corollary [5.4 and (20). 
Finally, observing that the integrals below converge absolutely and interpreting the limits as limits in the $L^{2}$-norm,

$$
\begin{aligned}
\mathcal{F}\left(\Lambda_{a} f\right)(\xi, j) & =\lim _{\substack{\varepsilon \rightarrow 0^{+} \\
R \rightarrow+\infty}} \mathcal{F}\left(\Lambda_{a,(\varepsilon, R)} f\right)(\xi, j) \\
& =\lim _{\substack{\varepsilon \rightarrow 0^{+} \\
R \rightarrow+\infty}} \int_{\varepsilon<\left|\frac{\pi t}{2 a}\right|<R} \frac{e^{-\frac{t}{2}}}{\sinh \left(\frac{\pi t}{2 a}\right)} \int_{\mathbb{R}} \chi_{+}(x) \mathcal{F}_{2} f\left(x e^{-t}, j\right) e^{-i x \xi} d x d t \\
& =\lim _{\substack{\varepsilon \rightarrow 0^{+} \\
R \rightarrow+\infty}} \int_{\varepsilon<\left|\frac{\pi t}{2 a}\right|<R} \frac{e^{-\frac{t}{2}}}{\sinh \left(\frac{\pi t}{2 a}\right)} \mathcal{F}\left(\left(\chi_{+} f\right)\left((\cdot) e^{-t}, \cdot\right)\right)(\xi, j) d t \\
& =\lim _{\substack{\varepsilon \rightarrow 0^{+} \\
R \rightarrow+\infty}} \int_{\varepsilon<\left|\frac{\pi t}{2 a}\right|<R} \frac{e^{\frac{t}{2}}}{\sinh \left(\frac{\pi t}{2 a}\right)} \mathcal{F}\left(\chi_{+} f\right)\left(\xi e^{t}, j\right) d t .
\end{aligned}
$$

Suppose now that $\xi>0$. Then,

$$
\begin{aligned}
\int_{\varepsilon<\left|\frac{\pi t}{2 a}\right|<R} \frac{e^{\frac{t}{2}}}{\sinh \left(\frac{\pi t}{2 a}\right)} \mathcal{F}\left(\chi_{+} f\right)\left(\xi e^{t}, j\right) d t & =e^{-\frac{\log \xi}{2}} \int_{\varepsilon<\left|\frac{\pi t}{2 a}\right|<R} \frac{e^{\frac{\log \xi+t}{2}}}{\sinh \left(\frac{\pi t}{2 a}\right)} \mathcal{F}\left(\chi_{+} f\right)\left(e^{\log \xi+t}, j\right) d t \\
& =\Lambda_{a,(\varepsilon, R)}\left(\mathcal{F}\left(\chi_{+} f\right)\right)(\xi, j) .
\end{aligned}
$$

Therefore, if $\xi>0$,

$$
\mathcal{F}\left(\Lambda_{a} f\right)(\xi, j)=\Lambda_{a}\left(\mathcal{F}\left(\chi_{+} f\right)\right)(\xi, j),
$$

as we wished to show. Similarly, if $\xi<0$, we get

$$
\begin{aligned}
\int_{\varepsilon<\left|\frac{\pi t}{2 a}\right|<R} \frac{e^{\frac{t}{2}}}{\sinh \left(\frac{\pi t}{2 a}\right)} \mathcal{F}\left(\chi_{+} f\right)\left(\xi e^{t}, j\right) d t & =e^{-\frac{\log (-\xi)}{2}} \int_{\varepsilon<\left|\frac{\pi t}{2 a}\right|<R} \frac{e^{\frac{\log (-\xi)+t}{2}}}{\sinh \left(\frac{\pi t}{2 a}\right)} \mathcal{F}\left(\chi_{+} f\right)\left(-e^{\log (-\xi)+t}, j\right) d t \\
& =\Lambda_{a,(\varepsilon, R)}\left(\widehat{\mathcal{F}\left(\chi_{+} f\right)}\right)(\xi, j)
\end{aligned}
$$

and $(i v)$ is finally proved.

We now have all the ingredients to prove Theorem 5.2, The boundedness of $P_{a}$ will follow from the following lemma.

Lemma 5.6. Let be $|a| \geq \pi$. Then, for every $s \in\left[0, \frac{\pi}{2|a|}\right)$,

(i) the operator $\Lambda_{+}$defines a bounded operator $\Lambda_{+}: W^{s, 2}(\mathbb{R} \times \mathbb{T}) \rightarrow W^{s, 2}(\mathbb{R} \times \mathbb{T})$;

(ii) the operator $\Lambda_{a}$ defines a bounded operator $\Lambda_{a}: W^{s, 2}(\mathbb{R} \times \mathbb{T}) \rightarrow W^{s, 2}(\mathbb{R} \times \mathbb{T})$.

Before proving the lemma, we fix some notation and recall the definition of the classical Hilbert transform. We refer the reader, for instance, to [Gra08, Chapter 4].

Let be $f: \mathbb{R} \times \mathbb{T} \rightarrow \mathbb{C}$ a test function. The Hilbert transform with respect to the first variable is defined by the formula

$$
\mathcal{F}_{1}\left(\mathcal{H}_{1} f\right)(\xi, \theta)=-i \operatorname{sgn}(\xi) \mathcal{F}_{1}(f)(\xi, \theta),
$$

where as usual, $\mathcal{F}_{1}$ denotes the Fourier transform with respect to the first variable.

If $p \in(0, \infty)$ and $w(x)$ is a non-negative real function, then we denote by $L^{p}(w)$ the space of functions such that

$$
\int_{\mathbb{R}}|g(x)|^{p} w(x) d x<\infty .
$$


It is a well-known result that the Hilbert transform extends to a bounded operator $L^{p}(w) \rightarrow$ $L^{p}(w)$ whenever $w$ is a weight in the Muckenhoupt class $A_{p}$ (see, e.g., Gra09]).

We are now ready to prove the lemma.

Proof of Lemma 5.6. In order to prove the boundedness of $\Lambda_{+}$, by Plancherel's formula, we need to estimate

$$
\left\|\Lambda_{+} f\right\|_{W^{s, 2}(\mathbb{R} \times \mathbb{T})}^{2}=\frac{1}{2 \pi} \sum_{j \in \mathbb{Z}} \int_{\mathbb{R}}\left(1+\xi^{2}+j^{2}\right)^{s}\left|\mathcal{F}\left(\chi_{+} f\right)(\xi, j)\right|^{2} d \xi .
$$

Notice that

$$
\begin{aligned}
\mathcal{F}\left(\chi_{+} f\right)(\xi, j) & =\frac{1}{2} \int_{-\infty}^{\infty}(1+\operatorname{sgn}(x)) \mathcal{F}_{2} f(x, j) e^{-i x \xi} d \xi \\
& =\frac{1}{2}\left(\mathcal{F} f(\xi, j)-i \mathcal{H}_{1} \mathcal{F} f(\xi, j)\right) .
\end{aligned}
$$

Moreover, since $s<\frac{\pi}{2|a|}<\frac{1}{2}$, it holds

$$
\left(1+\xi^{2}+j^{2}\right)^{s} \leq 1+\xi^{2 s}+j^{2 s}
$$

and the weight $w(\xi)=\xi^{2 s}$ turns out to belong to the $A_{2}$ class (see Gra09, Example 9.1.8]).

Therefore,

$$
\begin{aligned}
\left\|\chi_{+} f\right\|_{W^{s, 2}}^{2} & \leq \frac{1}{2 \pi} \sum_{j \in \mathbb{Z}} \int_{\mathbb{R}}\left(1+\xi^{2}+j^{2}\right)^{s}\left|\mathcal{F}\left(\chi_{+} f\right)(\xi, j)\right|^{2} d \xi \\
& \leq C \sum_{j \in \mathbb{Z}} \int_{\mathbb{R}}\left(1+\xi^{2}+j^{2}\right)^{s}\left|\mathcal{F} f(\xi, j)-i \mathcal{H}_{1} \mathcal{F} f(\xi, j)\right|^{2} d \xi \\
& \leq C\|f\|_{W^{s, 2}}^{2}+C \sum_{j \in \mathbb{Z}} \int_{\mathbb{R}}\left(1+\xi^{2 s}+j^{2 s}\right)\left|\mathcal{H}_{1} \mathcal{F} f(\xi, j)\right|^{2} d \xi
\end{aligned}
$$

From the boundedness of $\mathcal{H}_{1}$ in $L^{2}$, in $L^{2}\left(\xi^{2 s}\right)$ and exploiting also Plancherel's formula, we obtain

$$
\begin{aligned}
\sum_{j \in \mathbb{Z}} \int_{\mathbb{R}}\left(1+\xi^{2 s}+j^{2 s}\right)\left|\mathcal{H}_{1} \mathcal{F} f(\xi, j)\right|^{2} d \xi & \leq C \sum_{j \in \mathbb{Z}} \int_{\mathbb{R}}\left(1+\xi^{2 s}+j^{2 s}\right)|\mathcal{F} f(\xi, j)|^{2} d \xi \\
& \leq C \sum_{j \in \mathbb{Z}} \int_{\mathbb{R}}\left(1+\xi^{2}+j^{2}\right)^{s}|\mathcal{F} f(\xi, j)|^{2} d \xi \\
& \leq C\|f\|_{W^{s, 2}}^{2},
\end{aligned}
$$

as we wished to show.

Analogously, for the operator $\Lambda_{a}$ we have

$$
\begin{aligned}
\sum_{j \in \mathbb{Z}} \int_{\mathbb{R}}\left(1+\xi^{2}+j^{2}\right)^{s}\left|\mathcal{F}\left(\Lambda_{a} f\right)(\xi, j)\right|^{2} d \xi & \leq \sum_{j \in \mathbb{Z}} \int_{\mathbb{R}}\left(1+\xi^{2 s}+j^{2 s}\right)\left|\mathcal{F}\left(\Lambda_{a} f\right)(\xi, j)\right|^{2} d \xi \\
& \leq \sum_{j \in \mathbb{Z}}\left[\int_{0}^{\infty}+\int_{-\infty}^{0}\right]\left(1+\xi^{2 s}+j^{2 s}\right)\left|\mathcal{F}\left(\Lambda_{a} f\right)(\xi, j)\right|^{2} d \xi
\end{aligned}
$$


We now focus on the integration over the positive $\xi$ 's. From Corollary 5.5 (iv), for $\xi>0$, using the fact that $s<\frac{\pi}{2|a|}$ we have

$$
\begin{aligned}
\xi^{s} \mathcal{F}\left(\Lambda_{a} f\right)(\xi, j) & =\xi^{s} \Lambda_{a}\left(\mathcal{F}\left(\chi_{+} f\right)\right)(\xi, j) \\
& =\xi^{s} e^{-\frac{\log \xi}{2}} \lim _{\varepsilon \rightarrow 0^{+}} \int_{\varepsilon<\left|\frac{\pi t}{2 a}\right|} \frac{1}{\sinh \left(\frac{\pi t}{2 a}\right)} \mathcal{F}\left(\chi_{+} f\right)\left(e^{\log \xi-t}, j\right) e^{\frac{\log \xi-t}{2}} d t \\
& =e^{-\frac{\log \xi}{2}} \lim _{\varepsilon \rightarrow 0^{+}} \int_{\varepsilon<\left|\frac{\pi t}{2 a}\right|} \frac{e^{s t}}{\sinh \left(\frac{\pi t}{2 a}\right)} e^{s(\log \xi-t)} \mathcal{F}\left(\chi_{+} f\right)\left(e^{\log \xi-t}, j\right) e^{\frac{\log \xi-t}{2}} d t \\
& =e^{-\frac{\log \xi}{2}} \lim _{\varepsilon \rightarrow 0^{+}} \int_{\varepsilon<\left|\frac{\pi t}{2 a}\right|} \frac{e^{s t}}{\sinh \left(\frac{\pi t}{2 a}\right)} e^{s(\log \xi-t)} \mathcal{F}\left(\chi_{+} f\right)\left(e^{\log \xi-t}, j\right) e^{\frac{\log \xi-t}{2}} d t \\
& =\mathcal{C}_{2}^{-1} T_{a, s} \mathcal{C}_{2}\left((\cdot)^{s} \mathcal{F}\left(\chi_{+} f\right)\right)(\xi, j),
\end{aligned}
$$

where $\left((\cdot)^{s} \mathcal{F}\left(\chi_{+} f\right)\right)(\xi, j)=\xi^{s} \mathcal{F}\left(\chi_{+} f\right)(\xi, j)$. Therefore, from the $L^{2}$ boundedness of $T_{a, s}$ and $\Lambda_{+}$, we get

$$
\begin{aligned}
\sum_{j \in \mathbb{Z}} \int_{0}^{\infty} \xi^{2 s}\left|\mathcal{F}\left(\Lambda_{a} f\right)(\xi, j)\right|^{2} d \xi & \leq C \sum_{j \in \mathbb{Z}} \int_{\mathbb{R}}\left|\mathcal{C}_{2}\left((\cdot)^{s} \mathcal{F}\left(\chi_{+} f\right)\right)(\xi, j)\right|^{2} d \xi \\
& \leq C \sum_{j \in \mathbb{Z}} \int_{0}^{\infty} \xi^{2 s}\left|\mathcal{F}\left(\chi_{+} f\right)(\xi, j)\right|^{2} d \xi \\
& \leq\left. C \sum_{j \in \mathbb{Z}} \int_{\mathbb{R}}\left(1+\xi^{2}+j^{2}\right)^{s} \mathcal{F}\left(\chi_{+} f\right)(\xi, j)\right|^{2} d \xi \\
& \leq C\|f\|_{W^{s, 2}}^{2} .
\end{aligned}
$$

Similarly, from Corollary 5.5 and the $L^{2}$ boundedness of $\Lambda_{a}$ and $\Lambda_{+}$, we deduce

$$
\begin{aligned}
\sum_{j \in \mathbb{Z}}\left(1+j^{2 s}\right) \int_{0}^{\infty}\left|\mathcal{F}\left(\Lambda_{a} f\right)(\xi, j)\right|^{2} d \xi & =\sum_{j \in \mathbb{Z}}\left(1+j^{2 s}\right) \int_{0}^{\infty}\left|\Lambda_{a}\left(\mathcal{F}\left(\chi_{+} f\right)\right)(\xi, j)\right|^{2} d \xi \\
& \leq C \sum_{z \in \mathbb{Z}}\left(1+j^{2 s}\right) \int_{\mathbb{R}}\left|\mathcal{F}\left(\chi_{+} f\right)(\xi, j)\right|^{2} d \xi \\
& \leq\left. C \sum_{j \in \mathbb{Z}} \int_{\mathbb{R}}\left(1+\xi^{2}+j^{2}\right)^{s} \mathcal{F}\left(\chi_{+} f\right)(\xi, j)\right|^{2} d \xi \\
& \leq C\|f\|_{W^{s, 2}}^{2} .
\end{aligned}
$$

Hence, we finally estimated the integration over the positive $\xi$ 's in (24). A completly analogous argument allows us to estimate the over the negative $\xi$ 's. Therefore, the proof is concluded.

Proof of Theorem 5.2. It follows from Corollary 5.42 and Lemma 5.6.

In order to conclude the proof of Theorem 2, in analogy with Theorem 5.2, we prove the following.

Theorem 5.7. The operator $Q_{a}$ defines a bounded operator

$$
Q_{a}: W^{s, 2}(\mathbb{R} \times \mathbb{T}) \rightarrow W^{s, 2}(\mathbb{R} \times \mathbb{T})
$$

for every $s \in\left[0, \frac{\pi}{2|a|}\right)$. 
Proof. By density, we consider a test function $f$ on $\mathbb{R} \times \mathbb{T}$ which is a trigonometric polynomial in the second variable, that is,

$$
f(x, \theta)=\sum_{j=-N}^{N} f(x, j) e^{i j \theta} .
$$

Then, setting

$$
g_{j}(x, \theta):=x^{-i\left(\frac{j}{2}+\frac{1}{4}\right)}\left(\chi_{+} f\right)(x, \theta) .
$$

and using (ii) of Corollary [5.4, we see that

$$
\begin{aligned}
Q_{a} f(x, \theta) & =\frac{e^{\frac{a}{4}}}{(2 \pi)^{2}} \chi_{+}(x) e^{-\frac{\log x}{2}} \sum_{j=-N}^{N} e^{i j \theta} \int_{\mathbb{R}} \frac{e^{a\left(\xi-\frac{j}{2}-\frac{1}{4}\right)}}{2 \cosh \left(a\left(\xi-\frac{j}{2}-\frac{1}{4}\right)\right.} \mathcal{F}\left(\mathcal{C}_{2}\left(\chi_{+} f\right)\right)(\xi, j) e^{i(\log x) \xi} d \xi \\
& =\frac{e^{\frac{a}{4}}}{(2 \pi)^{2}} \chi_{+}(x) e^{-\frac{\log x}{2}} \sum_{j=-N}^{N} e^{i j \theta} \int_{\mathbb{R}} \frac{e^{a \xi}}{2 \cosh (a \xi)} \mathcal{F}\left(\mathcal{C}_{2}\left(\chi_{+} f\right)\right)\left(\xi+\frac{j}{2}+\frac{1}{4}, j\right) e^{i(\log x)\left(\xi+\frac{j}{2}+\frac{1}{4}\right)} d \xi \\
& =\frac{e^{\frac{a}{4}}}{(2 \pi)^{2}} \chi_{+}(x) e^{-\log x\left(\frac{1}{2}-\frac{i}{4}\right)} \sum_{j=-N}^{N} e^{i j\left(\theta+\frac{\log x}{2}\right)} \int_{\mathbb{R}} \frac{e^{a \xi}}{2 \cosh (a \xi)} \mathcal{F}\left(\mathcal{C}_{2} g_{j}\right)(\xi, j) e^{i(\log x) \xi} d \xi \\
& =\frac{e^{\frac{a}{4}}}{2 \pi} \chi_{+}(x) e^{-\log x\left(\frac{1}{2}-\frac{i}{4}\right)} \sum_{j=-N}^{N} e^{i j\left(\theta+\frac{\log x}{2}\right)} T_{m_{a}}\left[\mathcal{F}_{2}\left(\mathcal{C}_{2} g_{j}\right)(\cdot, j)\right](\log x) \\
& =\frac{e^{\frac{a}{4}}}{2} \Lambda_{+} f(x, \theta)+\frac{e^{\frac{a}{4}}}{8 \pi|a| i} \chi_{+}(x) e^{-\log x\left(\frac{1}{2}-\frac{i}{4}\right)} \sum_{j=-N}^{N} e^{i j\left(\theta+\frac{\log x}{2}\right)} T_{a, 0}\left(\mathcal{F}_{2} \mathcal{C}_{2} g_{j}\right)(\log x, j) \\
& =: C \Lambda_{+} f(x, \theta)+C^{\prime} \widetilde{\Lambda_{a}} f(x, \theta),
\end{aligned}
$$

where $C, C^{\prime}$ are positive constants.

Therefore, to conclude the proof we only have to prove boundedness of the operator $\widetilde{\Lambda_{a}}$. Arguing as in the proof of Corollary 5.5 (iv), for $\xi>0$ we get

$$
\begin{aligned}
\mathcal{F} \widetilde{\Lambda_{a}} f(\xi, j) & =\int_{\mathbb{R}} \chi_{+}(x) \lim _{\varepsilon \rightarrow 0} \int_{\left|\frac{\pi t}{2 a}\right|>\varepsilon} \frac{e^{i t\left(\frac{j}{2}+\frac{1}{4}\right)} e^{-\frac{t}{2}}}{\sinh \left(\frac{\pi t}{2 a}\right)} \mathcal{F}_{2}\left(\chi_{+} f\right)\left(x e^{-t}, j\right) d t e^{-i x \xi} d x \\
& =e^{-i\left(\frac{j}{2}+\frac{1}{4}\right) \log \xi} \mathcal{C}_{2}^{-1} T_{a, 0} \mathcal{C}_{2}\left((\cdot)^{i\left(\frac{j}{2}+\frac{1}{4}\right)} \mathcal{F}\left(\chi_{+} f\right)\right)(\xi, j) \\
& =e^{-i\left(\frac{j}{2}+\frac{1}{4}\right) \log \xi} \Lambda_{a}\left((\cdot)^{i\left(\frac{j}{2}+\frac{1}{4}\right)} \mathcal{F}\left(\chi_{+} f\right)\right)(\xi, j) .
\end{aligned}
$$


Therefore, similarly to (24), we obtain

$$
\begin{aligned}
\sum_{j \in \mathbb{Z}} \int_{0}^{\infty}\left(1+\xi^{2}+j^{2}\right)^{s}\left|\mathcal{F} \widetilde{\Lambda_{a}} f(\xi, j)\right|^{2} d \xi & =\left.\left.\sum_{j \in \mathbb{Z}} \int_{0}^{\infty}\left(1+\xi^{2}+j^{2}\right)\right|^{s} \Lambda_{a}\left((\cdot)^{i\left(\frac{j}{2}+\frac{1}{4}\right)} \mathcal{F}\left(\chi_{+} f\right)\right)(\xi, j)\right|^{2} d \xi \\
& \leq\left.\left.\sum_{j \in \mathbb{Z}} \int_{0}^{\infty}\left(1+\xi^{2}+j^{2}\right)\right|^{s}\left((\cdot)^{i\left(\frac{j}{2}+\frac{1}{4}\right)} \mathcal{F}\left(\chi_{+} f\right)\right)(\xi, j)\right|^{2} d \xi \\
& =\left.\left.\sum_{j \in \mathbb{Z}} \int_{0}^{\infty}\left(1+\xi^{2}+j^{2}\right)\right|^{s} \mathcal{F}\left(\chi_{+} f\right)(\xi, j)\right|^{2} d \xi \\
& \leq\|f\|_{W^{s, 2}(\mathbb{R} \times \mathbb{T})}^{2} .
\end{aligned}
$$

With a similar argument we estimate the integration over negative $\xi$ 's and we are done.

Finally, Theorems 5.2 and 5.7 imply the boundedness $\mathscr{P}_{k, \ell}: W^{s, 2}\left(d_{b}\left(D_{\beta}\right)\right) \rightarrow W^{s, 2}\left(d_{b}\left(D_{\beta}\right)\right)$, hence, the boundedness of the Szegö projection $\mathscr{P}$. The proof of Theorem 2 is complete.

\section{Proof of Theorem 3.3}

In order to conclude the proof of Theorem 3.3, we exploit the space $\mathscr{H}^{2}\left(D_{\beta}^{\prime}\right)$ studied in Mon16b. We recall here the main facts needed for our purposes and we refer the reader to Mon16b] for the proofs.

6.1. The Hardy space $\mathscr{H}^{2}\left(D_{\beta}^{\prime}\right)$. Consider the domain

$$
D_{\beta}^{\prime}=\left\{\left(z_{1}, z_{2}\right) \in \mathbb{C}^{2}:\left.\left|\operatorname{Im} z_{1}-\log \right| z_{2}\right|^{2}\left|<\frac{\pi}{2},\right| \log \left|z_{2}\right|^{2} \mid<\beta-\frac{\pi}{2}\right\}
$$

and its distinguished boundary

$$
d_{b}\left(D_{\beta}^{\prime}\right)=\left\{\left(z_{1}, z_{2}\right) \in \mathbb{C}^{2}:\left.\left|\operatorname{Im} z_{1}-\log \right| z_{2}\right|^{2}\left|=\frac{\pi}{2},\right| \log \left|z_{2}\right|^{2} \mid=\beta-\frac{\pi}{2}\right\} .
$$

Then, the space $\mathscr{H}^{2}\left(D_{\beta}^{\prime}\right)$ is defined as the function space

$$
\mathscr{H}^{2}\left(D_{\beta}^{\prime}\right)=\left\{f \in \operatorname{Hol}\left(D_{\beta}^{\prime}\right):\|f\|_{\mathscr{H}^{2}\left(D_{\beta}^{\prime}\right)}^{2}=\sup _{(t, s) \in\left[0, \frac{\pi}{2}\right) \times\left[0, \beta-\frac{\pi}{2}\right)}\|f\|_{L^{2}\left(d_{b}\left(D_{\beta}^{\prime}\right)\right)}^{2}<\infty\right\},
$$

where,

$$
D_{t, s}^{\prime}=\left\{\left(z_{1}, z_{2}\right) \in \mathbb{C}^{2}:\left.\left|\operatorname{Im} z_{1}-\log \right| z_{2}\right|^{2}|<t,| \log \left|z_{2}\right|^{2} \mid<s\right\},
$$

and

$$
\begin{aligned}
& \|F\|_{L^{2}\left(d_{b}\left(D_{\beta}^{\prime}\right)\right)}^{2}= \\
& \quad \int_{\mathbb{R}} \int_{0}^{2 \pi}\left|F\left(x+i(s+t), e^{\frac{s}{2}} e^{i \theta}\right)\right|^{2} e^{\frac{s}{2}} d \theta d x+\int_{\mathbb{R}} \int_{0}^{2 \pi}\left|F\left(x+i(s-t), e^{\frac{s}{2}} e^{i \theta}\right)\right|^{2} e^{\frac{s}{2}} d \theta d x \\
& \quad+\int_{\mathbb{R}} \int_{0}^{2 \pi}\left|F\left(x-i(s+t), e^{-\frac{s}{2}} e^{i \theta}\right)\right|^{2} e^{-\frac{s}{2}} d \theta d x+\int_{\mathbb{R}} \int_{0}^{2 \pi}\left|F\left(x-i(s-t), e^{-\frac{s}{2}} e^{i \theta}\right)\right|^{2} e^{-\frac{s}{2}} d \theta d x .
\end{aligned}
$$

We notice that

$$
d_{b}\left(D_{\beta}^{\prime}\right)=E_{1}^{\prime} \cup E_{2}^{\prime} \cup E_{3}^{\prime} \cup E_{4}^{\prime}
$$


where

$$
\begin{aligned}
& E_{1}^{\prime}=\left\{\left(z_{1}, z_{2}\right): \operatorname{Im} z_{1}=\beta, \log \left|z_{2}\right|^{2}=\beta-\frac{\pi}{2}\right\} \\
& E_{2}^{\prime}=\left\{\left(z_{1}, z_{2}\right): \operatorname{Im} z_{1}=\beta-\pi, \log \left|z_{2}\right|^{2}=\beta-\frac{\pi}{2}\right\} \\
& E_{3}^{\prime}=\left\{\left(z_{1}, z_{2}\right) \operatorname{Im} z_{1}=-\beta, \log \left|z_{2}\right|^{2}=-\beta+\frac{\pi}{2}\right\} ; \\
& E_{4}^{\prime}=\left\{\left(z_{1}, z_{2}\right): \operatorname{Im} z_{1}=-\beta+\pi, \log \left|z_{2}\right|^{2}=-\beta+\frac{\pi}{2}\right\}
\end{aligned}
$$

and each component $E_{\ell}^{\prime}, \ell=1, \ldots, 4$, can be identified with $\mathbb{R} \times \mathbb{T}$. Therefore, the restriction of any function defined on $d_{b}\left(D_{\beta}^{\prime}\right)$ to any of the component $E_{\ell}^{\prime}$ can be identified with a function defined on $\mathbb{R} \times \mathbb{T}$.

Denoting by $\chi_{\ell}^{\prime}$ the characteristic function of $E_{\ell}^{\prime}, \ell=1, \ldots, 4$, the Szegö projection $\mathscr{P}^{\prime} f$ of a function $f \in L^{2}\left(d_{b}\left(D_{\beta}^{\prime}\right)\right)$ is given by

$$
\mathscr{P}^{\prime} f=\sum_{k, \ell=1}^{4} \chi_{k}^{\prime} \mathscr{P}^{\prime}\left(\chi_{\ell}^{\prime} f\right),
$$

where, writing $\chi_{k}^{\prime} \mathscr{P}^{\prime}\left(\chi_{\ell}^{\prime} \cdot\right)=\mathscr{P}_{k, \ell}^{\prime}$ we adopt the convention that the operator $\mathscr{P}_{k, \ell}^{\prime}$ is acting on functions defined on $\mathbb{R} \times \mathbb{T}$.

Now, by [Mon16b, Remark 3.13] it follows that

$$
\mathscr{P}_{k, \ell}^{\prime}=\mathcal{F}^{-1}\left(\tilde{m}_{k, \ell} \mathcal{F}\right)=T_{\widetilde{m}_{k, \ell}},
$$

where

$$
\tilde{m}_{k, \ell}=m_{k, \ell}\left(\frac{1}{2}-i \cdot, \cdot\right)
$$

and for $k, \ell \in\{1, \ldots, 4\}, m_{k, \ell}$ are as in Def. 3.2.

Thus, the operator $\mathscr{P}^{\prime}$ is obtained as a sum of Fourier multiplier operators - and its mapping properties were studied in [Mon16b].

6.2. Relationship between $\mathscr{P}^{\prime}$ and $\mathscr{P}$. We now show that $\mathscr{H}^{2}\left(D_{\beta}\right)$ and $\mathscr{H}^{2}\left(D_{\beta}^{\prime}\right)$ are isometric and, as a consequence, we obtain a transformation rule for the Szegő projections $\mathscr{P}^{\prime}$ and $\mathscr{P}$.

The domains $D_{\beta}^{\prime}$ and $D_{\beta}$ are biholomorphic via the biholomorphism

$$
\begin{aligned}
\varphi: & D_{\beta}^{\prime} \rightarrow D_{\beta} \\
\left(z_{1}, z_{2}\right) \mapsto\left(e^{z_{1}}, z_{2}\right) & \left(z_{1}, z_{2}\right) \mapsto\left(\log \left(z_{1} e^{-i \log \left|z_{2}\right|^{2}}\right)+i \log \left|z_{2}\right|^{2}, z_{2}\right)
\end{aligned}
$$

where Log denotes the principal branch of the complex logarithm. It is straightforward to see that

$$
d_{b}\left(D_{\beta}\right)=\varphi\left(d_{b}\left(D_{\beta}^{\prime}\right)\right) \cup\left\{\left(0, z_{2}\right):\left.|\log | z_{2}\right|^{2} \mid=\beta-\frac{\pi}{2}\right\} .
$$

The following proposition holds.

Proposition 6.1. For $1<p<\infty$ be fixed. Let $\psi_{p}$ be given by

$$
\psi_{p}\left(z_{1}, z_{2}\right):=e^{-\frac{i}{p} \log \left|z_{2}\right|^{2}}\left(z_{1} e^{-i \log \left|z_{2}\right|^{2}}\right)^{-\frac{1}{p}} .
$$

Let $\varphi^{-1}$ be as in (26) and define the operator

$$
\Lambda f:=\psi_{p}\left(f \circ \varphi^{-1}\right) .
$$


Then, $\psi_{p} \in \operatorname{Hol}\left(D_{\beta}^{\prime}\right)$ and

$$
\Lambda: \mathscr{H}^{p}\left(D_{\beta}^{\prime}\right) \rightarrow \mathscr{H}^{p}\left(D_{\beta}\right)
$$

is a surjective isometry.

Proof. It is easy to see that $\psi_{p} \in \operatorname{Hol}\left(D_{\beta}^{\prime}\right)$ (or see [KPS16, Lemma 1.2]). The holomorphicity of $\Lambda f$ on $D_{\beta}$ follows immediately from the holomorphicity of $\varphi^{-1}$ and $\psi_{p}$, whereas the equality $\|f\|_{\mathscr{H}^{p}\left(D_{\beta}^{\prime}\right)}=\|\Lambda f\|_{\mathscr{H}^{p}\left(D_{\beta}\right)}$ follows at once from the fact that $\|f\|_{L^{p}\left(d_{b}\left(D_{t, s}^{\prime}\right)\right)}=\|\Lambda f\|_{L^{p}\left(d_{b}\left(D_{t, s}\right)\right)}$ for every $(t, s) \in\left(0, \frac{\pi}{2}\right) \times\left[0, \beta-\frac{\pi}{2}\right)$, as can be seen by a simple change of variables.

We now notice that this proposition implies part (ii) in Proposition 3.1. For, let $F \in H^{p}\left(D_{\beta}\right)$ and set $f=\Lambda^{-1} F \in H^{p}\left(D_{\beta}^{\prime}\right)$. By [Mon16b, Thm. 4.3] we know that the analogous conclusion holds true for $f$. Using part(i), (ii) in Proposition 3.1 now follows easily.

Using the proposition, we obtain a transformation rule for the Szegö kernels and projections of the spaces $\mathscr{H}^{2}\left(D_{\beta}\right)$ and $\mathscr{H}^{2}\left(D_{\beta}^{\prime}\right)$ similar to the one proved by Bell in [Bel92, Thm. 12.3] for smooth bounded domains in $\mathbb{C}$. We leave the elementary details to the reader.

Proposition 6.2. Let $K$ and $K^{\prime}$ be the reproducing kernels of $\mathscr{H}^{2}\left(D_{\beta}\right)$ and $\mathscr{H}^{2}\left(D_{\beta}^{\prime}\right)$ respectively. Then,

$$
K\left(\left(z_{1}, z_{2}\right),\left(w_{1}, w_{2}\right)\right)=\psi_{2}\left(z_{1}, z_{2}\right) K^{\prime}\left(\varphi^{-1}\left(z_{1}, z_{2}\right), \varphi^{-1}\left(w_{1}, w_{2}\right)\right) \overline{\psi_{2}\left(w_{1}, w_{2}\right)} .
$$

Hence, if $\mathscr{P}^{\prime}$ and $\mathscr{P}$ are the Szegö projections of $\mathscr{H}^{2}\left(D_{\beta}^{\prime}\right)$ and $\mathscr{H}^{2}\left(D_{\beta}\right)$ respectively and $f \in$ $L^{2}\left(d_{b}\left(D_{\beta}\right)\right)$, then

$$
\mathscr{P}^{\prime}\left(\Lambda^{-1} f\right)=\Lambda^{-1}(\mathscr{P} f)
$$

Finally, we have,

End of proof of Theorem 3.3. From Proposition 6.2 we have that

$$
\mathscr{P} f=\sum_{k, \ell=1}^{4} \mathscr{P}_{k, \ell} f=\sum_{k, \ell=1}^{4} \chi_{k} \mathscr{P}\left(\chi_{\ell} f\right)=\sum_{k, \ell=1}^{4} \chi_{k} \psi_{2} \cdot \mathscr{P}^{\prime}\left(\left(\frac{1}{\psi_{2}} \chi_{\ell} f\right) \circ \varphi\right) \circ \varphi^{-1} .
$$

Observe that

$$
\mathscr{P}^{\prime}\left(\left(\frac{1}{\psi_{2}} \chi_{\ell} f\right) \circ \varphi\right)=\sum_{k, m=1}^{4} \chi_{k}^{\prime} \mathscr{P}^{\prime}\left(\chi_{m}^{\prime} \cdot\left(\frac{1}{\psi_{2}} \chi_{\ell} f\right) \circ \varphi\right)=\sum_{k=1}^{4} \chi_{k}^{\prime} \mathscr{P}^{\prime}\left(\chi_{\ell}^{\prime} \cdot\left(\frac{1}{\psi_{2}} \chi_{\ell} f\right) \circ \varphi\right),
$$

since $\chi_{\ell} \circ \varphi=\chi_{\ell}^{\prime}$, so it follows that $\left.\chi_{m}^{\prime} \cdot\left(\frac{1}{\psi_{2}} \chi_{\ell} f\right) \circ \varphi\right)$ does not vanish identically only if $m=\ell$. Therefore, using (25)

$$
\mathscr{P}^{\prime}\left(\left(\frac{1}{\psi_{2}} \chi_{\ell} f\right) \circ \varphi\right)=\sum_{k=1}^{4} \mathscr{P}_{k, \ell}^{\prime}\left(\left(\frac{1}{\psi_{2}} \chi_{\ell} f\right) \circ \varphi\right)=\sum_{k=1}^{4} T_{\widetilde{m}_{k, \ell}}\left(\left(\frac{1}{\psi_{2}} \chi_{\ell} f\right) \circ \varphi\right)
$$

where $T_{\widetilde{m}_{k, \ell}}$ denotes the Fourier multiplier operator associated to $\widetilde{m}_{k, \ell}$ as in Section 2 ,

It is now an easy observation that

$$
\left(\frac{1}{\psi_{2}} \chi_{\ell} f\right) \circ \varphi=\mathcal{C}_{2}\left(\chi_{\ell} f\right)
$$


hence, from (27), the results in Section 2 and the first part of Theorem 3.3, we conclude that

$$
\mathscr{P} f=\sum_{k, \ell=1}^{4} \mathcal{C}_{2}^{-1} T_{\widetilde{m}_{k, \ell}} \mathcal{C}_{2} f=\sum_{k, \ell=1}^{4} \mathscr{T}_{m_{k, \ell}} f
$$

as we wished. This concludes the proof of the theorem.

\section{FinAL REMARKS}

As we mentioned, our final goal is to prove the (ir-)regularity of the Szeo" projection on the smooth, bounded, worm domain $\Omega_{\beta}$. In this case, there is no ambiguity in the definition of the (classical) space $H^{2}\left(\Omega_{\beta}\right)$ and the associated Szegö projection on the topological boundary. On the other hand, due to the nature of the domain $D_{\beta}$, and of its biholomorphic copy $D_{\beta}^{\prime}$, it was a natural choice to define and study the Szegö projection on the distinguished boundary. The drawback of this choice is that it is more complicated to transfer information from the Hardy space $\mathscr{H}^{2}\left(d_{b}\left(D_{\beta}\right)\right)$ to $H^{2}\left(b \Omega_{\beta}\right)$. We do not exclude that to fully understand the behavior of the Szegö projection $P_{\Omega_{\beta}}$ on the topological boundary $b \Omega_{\beta}$ it might be necessary to study the Hardy spaces on the topological boundary of $D_{\beta}$. The Hardy spaces on the topological boundary of $D_{\beta}^{\prime}$ have already been studied by the same authors in [MP16].

Finally, we remark that regularity of the Szegó projection, at least in a certain setting, is equivalent to the regularity of the Complex Green operator [HPR15]. Therefore, the (ir-)regularity of the Szegö projection $P_{\Omega_{\beta}}$ will also provided information about the (ir-)regularity of the Complex Green operator on $b \Omega_{\beta}$. We plan to come back to these questions in future works.

\section{REFERENCES}

[Bar92] D. E. Barrett, Behavior of the Bergman projection on the Diederich-Fornass worm, Acta Math. 168 (1992), no. 1-2, 1-10. MR 1149863 (93c:32033)

[BB95] D. Békollé and A. Bonami, Estimates for the Bergman and Szegö projections in two symmetric domains of $\mathbf{C}^{n}$, Colloq. Math. 68 (1995), no. 1, 81-100. MR 1311766

[BCS88] H. P. Boas, So-Chin Chen, and E. J. Straube, Exact regularity of the Bergman and Szegő projections on domains with partially transverse symmetries, Manuscripta Math. 62 (1988), no. 4, 467-475. MR 971689 (90a:32032)

[Bel92] S. R. Bell, The Cauchy transform, potential theory, and conformal mapping, Studies in Advanced Mathematics, CRC Press, Boca Raton, FL, 1992. MR 1228442 (94k:30013)

[BEP15] D. E. Barrett, D. Ehsani, and M. M. Peloso, Regularity of projection operators attached to worm domains, Doc. Math. 20 (2015), 1207-1225. MR 3424478

[BL14] D. E. Barrett and L. Lee, On the Szegö metric, J. Geom. Anal. 24 (2014), no. 1, 104-117. MR 3145917

[Boa85] H. P. Boas, Regularity of the Szegö projection in weakly pseudoconvex domains, Indiana Univ. Math. J. 34 (1985), no. 1, 217-223. MR 773403 (86g:32040)

[Boa87] The Szegö projection: Sobolev estimates in regular domains, Trans. Amer. Math. Soc. 300 (1987), no. 1, 109-132. MR 871667 (88d:32030)

[BS89] H. P. Boas and E. J. Straube, Complete Hartogs domains in $\mathbf{C}^{2}$ have regular Bergman and Szegó projections, Math. Z. 201 (1989), no. 3, 441-454. MR 999739 (90h:32052)

[BS91] Sobolev estimates for the complex Green operator on a class of weakly pseudoconvex boundaries, Comm. Partial Differential Equations 16 (1991), no. 10, 1573-1582. MR 1133741 (92m:35190)

[BŞ12] D. E. Barrett and S. Şahutoğlu, Irregularity of the Bergman projection on worm domains in $\mathbb{C}^{n}$, Michigan Math. J. 61 (2012), no. 1, 187-198. MR 2904008

[Chr96] M. Christ, Global $C^{\infty}$ irregularity of the $\bar{\partial}-$ Neumann problem for worm domains, J. Amer. Math. Soc. 9 (1996), no. 4, 1171-1185. MR 1370592 (96m:32014) 
[CŞ15] Z. Cuckovic and S. Şahutoğlu, Essential norm estimates for the $\bar{\partial}-$ Neumann operator on convex domains and worm domains, ArXiv e-prints (2015).

[DF77] K. Diederich and J. E. Fornaess, Pseudoconvex domains: an example with nontrivial Nebenhülle, Math. Ann. 225 (1977), no. 3, 275-292. MR 0430315 (55 \#3320)

[Duo01] J. Duoandikoetxea, Fourier analysis, Graduate Studies in Mathematics, vol. 29, American Mathematical Society, Providence, RI, 2001, Translated and revised from the 1995 Spanish original by David Cruz-Uribe. MR 1800316

[Gra08] L. Grafakos, Classical Fourier analysis, second ed., Graduate Texts in Mathematics, vol. 249, Springer, New York, 2008. MR 2445437 (2011c:42001)

[Gra09] _ Modern Fourier analysis, second ed., Graduate Texts in Mathematics, vol. 250, Springer, New York, 2009. MR 2463316 (2011d:42001)

[HPR15] P. S. Harrington, M. M. Peloso, and A. S. Raich, Regularity equivalence of the Szegö projection and the complex Green operator, Proc. Amer. Math. Soc. 143 (2015), no. 1, 353-367. MR 3272760

[KP08a] S. G. Krantz and M. M. Peloso, Analysis and geometry on worm domains, J. Geom. Anal. 18 (2008), no. 2, 478-510. MR 2393268 (2009d:32036)

[KP08b] The Bergman kernel and projection on non-smooth worm domains, Houston J. Math. 34 (2008), no. 3, 873-950. MR 2448387 (2010i:32032)

[KPS15] S. G. Krantz, M. M. Peloso, and C. Stoppato, Completeness on the worm domain and the Müntz-Szász problem for the Bergman space, ArXiv e-prints (2015).

[KPS16]_ B B Bergman kernel and projection on the unbounded worm domain, Ann. Sc. Norm. Pisa (to appear 2016).

[LS04] L. Lanzani and E. M. Stein, Szegö and Bergman projections on non-smooth planar domains, J. Geom. Anal. 14 (2004), no. 1, 63-86. MR 2030575 (2004m:30063)

[LS15a] _ Hardy spaces of holomorphic functions for domains in $\mathbb{C}^{n}$ with minimal smoothness, ArXiv e-prints (2015).

[LS15b] ㄴ The Cauchy-Szegö projection for domains in $\mathbb{C}^{n}$ with minimal smoothness, ArXiv e-prints (2015).

[Mon16a] A. Monguzzi, A Comparison Between the Bergman and Szegö Kernels of the Non-smooth Worm Domain $D_{\beta}^{\prime}$, Complex Anal. Oper. Theory 10 (2016), no. 5, 1017-1043. MR 3506304

[Mon16b] Hardy spaces and the Szegö projection of the non-smooth worm domain $D_{\beta}^{\prime}$, J. Math. Anal. Appl. 436 (2016), no. 1, 439-466. MR 3440104

[Mon16c] _ On Hardy spaces on worm domains, Concr. Oper. 3 (2016), 29-42. MR 3491881

[MP16] A. Monguzzi and M. M. Peloso, Regularity of the Szegö projection on model worm domains, ArXiv e-prints (2016).

[MS97] J. D. McNeal and E. M. Stein, The Szegő projection on convex domains, Math. Z. 224 (1997), no. 4, 519-553. MR 1452048 (98f:32023)

[MZ15] S. Munasinghe and Y. E. Zeytuncu, Irregularity of the Szegö projection on bounded pseudoconvex domains in $\mathbb{C}^{2}$, Integral Equations Operator Theory 82 (2015), no. 3, 417-422. MR 3355787

[NRSW89] A. Nagel, J.-P. Rosay, E. M. Stein, and S. Wainger, Estimates for the Bergman and Szegő kernels in $\mathbf{C}^{2}$, Ann. of Math. (2) 129 (1989), no. 1, 113-149. MR 979602 (90g:32028)

[PS77] D. H. Phong and E. M. Stein, Estimates for the Bergman and Szegö projections on strongly pseudoconvex domains, Duke Math. J. 44 (1977), no. 3, 695-704. MR 0450623 (56 \#8916)

[Roo85] P. G. Rooney, A survey of Mellin multipliers, Fractional calculus (Glasgow, 1984), Res. Notes in Math., vol. 138, Pitman, Boston, MA, 1985, pp. 176-187. MR 860095 (87j:42034)

[Sed75] A. M. Sedleckiur, An equivalent definition of the $H^{p}$ spaces in the half-plane, and some applications, Mat. Sb. (N.S.) 96(138) (1975), 75-82, 167. MR 0369703 (51 \#5935)

[Ste70] E. M. Stein, Boundary values of holomorphic functions, Bull. Amer. Math. Soc. 76 (1970), $1292-1296$. MR 0273055 (42 \#7936)

[Str86] E. J. Straube, Exact regularity of Bergman, Szegö and Sobolev space projections in nonpseudoconvex domains, Math. Z. 192 (1986), no. 1, 117-128. MR 835396 (87k:32045)

[Tri83] H. Triebel, Theory of function spaces, Monographs in Mathematics, vol. 78, Birkhäuser Verlag, Basel, 1983. MR 781540 (86j:46026) 
Dipartimento di Matematica "F. Enriques", Università degli Studi di Milano, Via C. Saldini 50, I-20133 MiLANO

E-mail address: alessandro.monguzzi@unimi.it

E-mail address: marco.peloso@unimi.it 